\title{
Análisis paleosísmico de la falla El Malpaso - El Salto, norte de Michoacán, México
}

\author{
Paleoseismological analysis of EI Malpaso - El Salto fault, north Michoacán, Mexico
}

Diana Cinthia Soria-Caballero ${ }^{1, *}$, Daniel Alberto Gómez-Calderón², Víctor Hugo Garduño-Monroy ${ }^{3, \dagger}$

${ }^{1}$ Escuela Nacional de Estudios Superiores, unidad Morelia, Universidad Nacional Autónoma de México, Antigua Carretera a Pátzcuaro no. 8701, Ex-Hacienda de San José de La Huerta, 58190, Morelia, Michoacán, México.

${ }^{2}$ Posgrado en Ciencias de la Tierra, Escuela Nacional de Estudios Superiores, unidad Morelia, Universidad Nacional Autónoma de México, Antigua Carretera a Pátzcuaro no. 8701, Ex-Hacienda de San José de La Huerta, 58190, Morelia, Michoacán, México.

${ }^{3}$ Instituto de Investigaciones en Ciencias de la Tierra, Universidad Michoacana de San Nicolás de Hidalgo, Avenida Francisco J. Mújica s/n, 58030, Morelia, Michoacán, México.

* Autor para correspondencia: (D. Soria-Caballero)dcinthiasoriac@gmail.com

\section{Cómo citar este artículo:}

Soria-Caballero, D. C., Gómez-Calderón, D. A., Garduño-Monroy, V.H., 2021, Análisis paleosísmico de la falla El Malpaso - El Salto, norte de Michoacán, México: Boletín de la Sociedad Geológica Mexicana, 73 (2), A181220. http://dx.doi.org/10.18268/ BSGM2021v73n2a181220

Manuscrito recibido: 31 de Agosto de 2020 Manuscrito corregido: 15 de Diciembre de 2020 Manuscrito aceptado: 27 de Diciembre de 2020

La revisión por pares es responsabilidad de la Universidad Nacional Autónoma de México.

\section{RESUMEN}

La falla El Malpaso-El Salto es una estructura con geometría en échelon, compuesta por seis segmentos principales que combinados alcanzan una longitud total de $28 \mathrm{~km}$ y que pertenece a la porción occidental del Sistema de Fallas Morelia-Acambay. Esta falla afecta unidades geológicas de edades variables, desde $18 \mathrm{Ma}$ en la zona sur del lago de Cuitzeo hasta 1.5 Ma en la región norte del Complejo Volcánico del Tzirate. La expresión de esta falla en el paisaje y la reciente ocurrencia de sismos de magnitud moderada en la zona indican que puede tener actividad tectónica importante. El análisis paleosísmico preliminar realizado en este trabajo permitió estimar que la falla El Malpaso-El Salto presenta tasas de deslizamiento a largo plazo (slip rate geológico) de 0.086 $\pm 0.015 \mathrm{~mm} / \mathrm{año}$, con períodos de recurrencia de $10021 \pm 1221$ años, produciendo desplazamientos verticales por evento de entre 80 y $100 \mathrm{~cm}$. De acuerdo a las relaciones empíricas resueltas para esta falla, si sufriera una ruptura en la totalidad de su longitud, tendría la capacidad de producir eventos con magnitudes máximas $M_{\mathrm{w}}$ en el rango de 6.6 a 7.3, lo cual es similar a las magnitudes máximas reportadas para otras fallas del Sistema de Fallas Morelia-Acambay. En la zona, la falla El Malpaso-El Salto posee características geométricas, dimensiones, dirección, tipo de movimiento y potencialidad muy similares a las de una falla paralela ubicada a menos de $3 \mathrm{~km}$ al sureste y que es conocida como la falla La Alberca-Teremendo. Ambas fallas tienen una orientación favorable respecto al campo de esfuerzos actual (extensión NO-SE) y sus geometrías lístricas sugieren que podrían interaccionar entre ellas durante un evento de ruptura, modificando las estimaciones de recurrencia y magnitud máxima esperada. Las interacciones entre fallas incrementan la peligrosidad del sistema, por lo que su estudio es especialmente importante para las evaluaciones de riesgo sísmico del centro de México. En el sector oeste del Sistema de Fallas Morelia-Acambay son necesarios más estudios para refinar las estimaciones de actividad de las fallas y comprender su comportamiento conjunto.

Palabras clave: Paleosismología, falla El Malpaso-El Salto, Sistema de Fallas Morelia-Acambay, peligrosidad sísmica.

\section{ABSTRACT}

The El Malpaso - El Salto fault is a structure with en échelon geometry, composed of six main segments which combined has a length of 28 $\mathrm{km}$. This fault belongs to the western sector of the Morelia-Acambay Fault System and affects Miocene - Pleistocene volcanic deposits related to the Cuitzeo ignimbrite and El Tzirate Volcanic Complex (18 and $<1.5 \mathrm{Ma}$, respectively). The landscape expression of this fault and the recent occurrence of earthquakes of moderate magnitude in the zone indicate that the studied fault could have important tectonic activity. The paleoseismic analysis of this work led us to estimate long-term slip rates (geologic SR) of $0.086 \pm 0.015 \mathrm{~mm} /$ year and recurrence intervals of $10021 \pm 1221$ years, considering a total vertical displacement between 80 and $100 \mathrm{~cm}$. According to empirical relationships resolved for the MASF, if the fault broke along its entive length, it would have the capacity to produce events with maximum magnitudes $\left(M_{W}\right)$ of 6.6 to 7.3 , similar to the maximum magnitude reported for other faults in the Morelia-Acambay Fault System. In the study zone, the El Malpaso-El Salto fault has analogous characteristics (such as geometry, dimensions, direction, movement and potentiality) to a neighbor parallel fault named $\mathrm{La}$ Alberca-Teremendo fault, located $3 \mathrm{~km}$ southeast of the El Malpaso-El Salto fault trace. Both faults are favorably oriented to the current stress field (NW$S E$ extension) and their listric geometries suggest a probable interaction between them during a rupture event. The interaction with other faults of the same system could modify the estimated recurrence interval and maximum expected magnitude; also faults interactions increase the fault's hazard, therefore its study is important for the seismic risk assessment in central México. Also, more detailed studies are necessary to evaluate the activity of the western part of the Morelia Acambay Fault System.

Keywords: Paleoseismology, El Malpaso-El Salto fault, Morelia-Acambay Fault System, seismic hazard. 


\section{Introducción}

El estudio de las fallas corticales del centro de México es fundamental para la estimación del riesgo sísmico del país. En el estado de Michoacán el fallamiento cortical más activo pertenece a la porción occidental del Sistema de Fallas Morelia-Acambay (SFMA), el Sistema de Fallas Zacapu-Querétaro (SFZQ Quintero-Legorreta, 2002; también llamado Zona de Fallas Zacapu-Salvatierra por Suter, 2002) y estructuras paralelas al sistema de fallas Taxco-San Miguel de Allende (SFTSMA) (Figura 1).

El SFMA ha sido estudiado desde el enfoque paleosismológico debido a sus antecedentes de sismicidad cortical histórica de magnitud significativa. En la región de Morelia y Cuitzeo, este sistema se manifiesta en el paisaje a través de escarpes evidentes con desplazamientos acumulados de centenas de metros y alineamientos volcánicos (p.e. Queréndaro y Complejo Volcánico El Tzirate, Figura 2; Gómez-Vasconcelos et al., 2020 y Pérez-Orozco et al., 2018, respectivamente).

En esta zona, la evaluación del potencial sísmico se complica debido a la presencia de actividad volcánica durante el Pleistoceno y Holoceno, las limitadas vías de acceso y las restricciones de permiso para trabajar en el área. Por ello, el análisis de las deformaciones geomorfológicas se vuelve fundamental para alcanzar una estimación confiable.

En enero del 2020, el Servicio Sismológico Nacional (wrwre.ssn.unam.mx) reportó en esta región la ocurrencia de seis sismos con magnitudes coda (mc) entre 3.7 y 3.9 y epicentros cercanos a las fallas del SFMA (falla Tarímbaro y falla La Alberca-Teremendo), lo cual sugiere que esta zona es sísmicamente activa y dicha actividad podría afectar significativamente las ciudades del norte de Michoacán y estados aledaños.

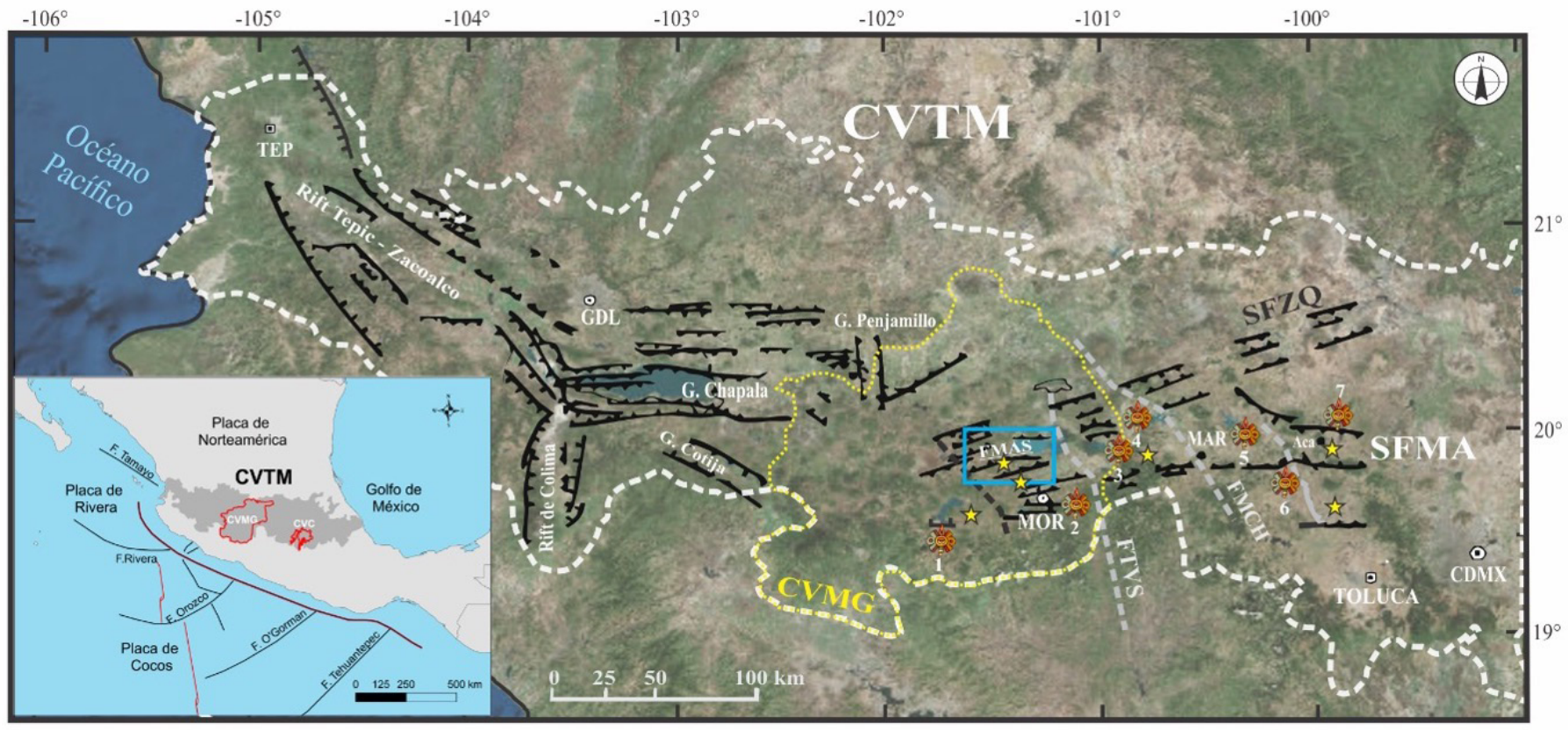

Figura 1 Localización del Cinturón Volcánico Trans-Mexicano (CVTM), el Campo Volcánico Michoacán Guanajuato (CVMG) y las principales estructuras tectónicas del centro-occidente de México. Símbolos: Estrellas amarillas = Estudios paleosísmicos previos; Ollin con número = Sismos históricos; Rectángulo azul = ubicación de figuras 2a y 2b; 1. Pátzcuaro, 1845 y 1858; 2. Morelia, 2007; 3. Araró, 1845; 4. UcareoZinapécuaro-Acámbaro, 1872 y 1874; 5. Maravatío, 1979; 6. Venta de Bravo, 1734-1755 y 1853-1854; 7. Acambay, 1912. Abreviaciones. SFMA: Sistema de Fallas Morelia-Acambay; SFZQ: Sistema de Fallas Zacapu-Querétaro; FTVS: Falla Tzitzio-Valle de Santiago; FMCH: Falla Maravatío - Ciudad Hidalgo; FMAS: Falla Malpaso - El Salto; GDL: Guadalajara; TEP: Tepic; MOR: Morelia; MAR: Maravatío; CDMX: Ciudad de México. *Ollin. Símbolo náhuatl que denota movimiento (Modificada de Soria-Caballero et al., 2019). 
La falla El Malpaso - El Salto (FMAS) es un segmento perteneciente al SFMA que posee características que sugieren un elevado potencial sísmico (escarpes lineares bien marcados, longitudes mayores a $10 \mathrm{~km}$ y geometría en échelon), sin embargo, carece de estudios que detallen su actividad. Cerca de esta falla ocurrió un sismo de $\mathrm{mc}=3.8$ a $5 \mathrm{~km}$ de profundidad, el 18 de enero de 2020, seguido por un sismo de mc $=3.7$ el 23 de Enero de 2020 e hipocentro a $15 \mathrm{~km}$ de profundidad (fuente: wrerr.ssn. unam.mx); aunque son eventos de magnitudes bajas y que deben ser sometidos a correcciones de localización y magnitud, son indicadores importantes de actividad. Considerando lo anterior, en este trabajo se realizó una primera aproximación a la evaluación de la actividad sísmica de esta falla mediante un análisis geomorfológico y paleosismológico, con el cual se pretende contribuir al conocimiento y la caracterización de las fallas activas del estado de Michoacán, así como del centro de México.

\section{Contexto geológico}

El SFMA es una franja de fallamiento cortical de $\sim 220 \mathrm{~km}$ de largo y $\sim 40 \mathrm{~km}$ de ancho, con dirección preferente ENE. Este sistema coexiste e interacciona con estructuras de primer orden que presentan orientaciones NE, las cuales pertenecen al tren de deformación o sistema de fallas Zacapu-Querétaro (Quintero-Legorreta, 2002), también nombrado zona de fallas Zacapu-Salvatierra (Suter et al., 2001 y Suter, 2002; Figura 1). El SFMA se encuentra en la zona más extensamente poblada de México, afectando el noreste del estado de Michoacán, el norte del Estado de México y el suroeste del estado de Hidalgo, los cuales poseen una población combinada de más de 15 millones de habitantes (wrere.inegi.org.mx); lo anterior incrementa la peligrosidad sísmica de los segmentos que lo conforman y, consecuentemente, la necesidad de caracterizar su actividad con detalle.

Este sistema cuenta con registro histórico e instrumental de sismos intraplaca con magnitudes tan altas como $M_{\mathrm{W}} 6.9$ (Acambay, 1912) o 7.2 (Ameca, 1567) (Urbina y Camacho, 1913; Suter, 2015, respectivamente) y los trabajos paleosísmicos publicados para las estructuras de este sistema muestran que ha tenido rupturas sísmicas recurrentes durante el Pleistoceno Superior y Holoceno (Langridge et al., 2000; Garduño-Monroy et al., 2009; Singh et al., 2012; Ortuño et al., 2015; Sunye-Puchol et al., 2015; Suter, 2016; Lacan et al., 2018; Velázquez-Bucio y Garduño-Monroy, 2018; Mendoza-Ponce et al., 2018; Ortuño et al., 2019; Soria-Caballero et al., 2019).
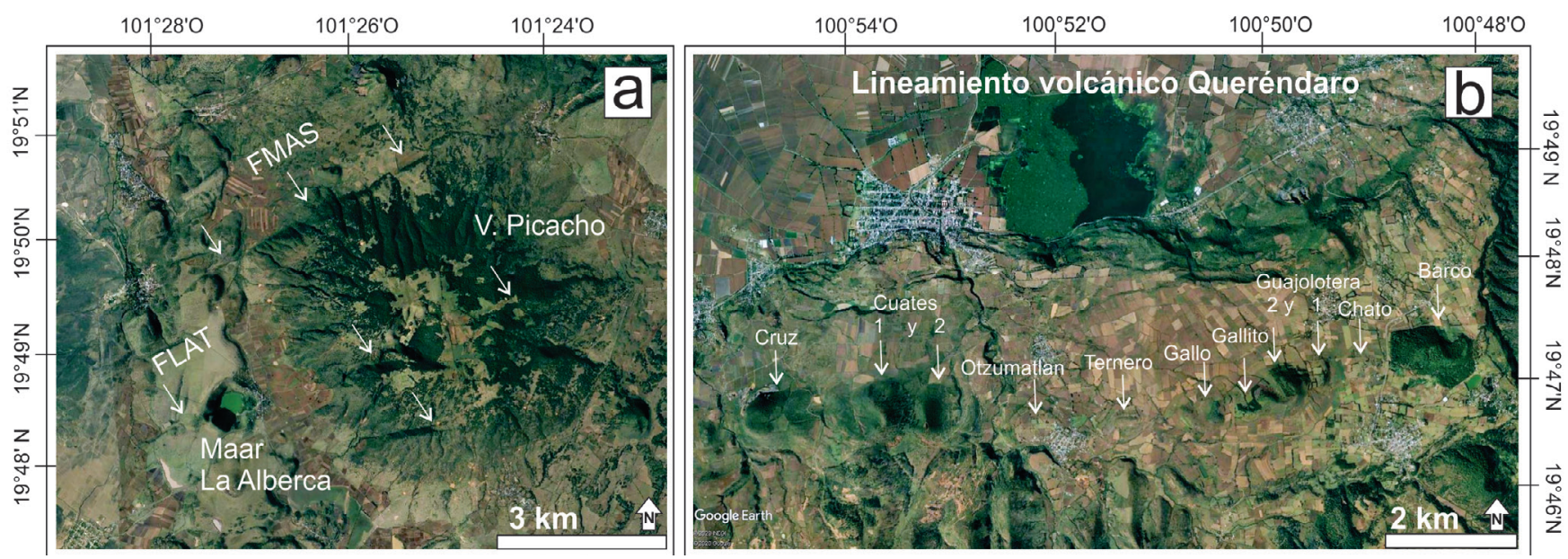

Figura 2 Ejemplos de la influencia de la actividad de las fallas del Sistema Morelia Acambay sobre los edificios y depósitos volcánicos del Campo Volcánico Michoacán-Guanajuato. Su ubicación se localiza en los rectángulos de la figura 1; a) fallas El Malpaso- El Salto (FMAS) y La Alberca-Teremendo (FLAT) cortando las laderas del volcán el Picacho, los flujos de lava del volcán Pelón y el mar La Alberca de Guadalupe; b) Alineamiento de conos volcánicos en Queréndaro relacionados con la falla Queréndaro. 
En el norte de Michoacán, las fallas del SFMA coexisten con los pulsos de actividad volcánica que dieron origen al Campo Volcánico Michoacán-Guanajuato (CVMG, una sub-provincia volcánica monogenética Cuaternaria de 40000 $\mathrm{km}^{2}$ ), por lo que muchas de las estructuras han perdido su expresión superficial primaria; sin embargo, su actividad y presencia puede observarse a través del alineamiento y dislocación de los edificios y depósitos volcánicos (Figura 2).

En esta zona, puede resultar complicado evaluar la actividad sísmica de las fallas del sistema debido a que la interacción entre los procesos volcánicos y tectónicos es tan estrecha, que puede guiar a sub- o sobreestimaciones de actividad y magnitud de sismos. No obstante, la marcada presencia en el paisaje de largas trazas de falla así como la existencia de zonas con afloramientos destacados, permite abordar el estudio de dichas fallas mediante el trabajo paleosismológico tradicional y evaluar la probabilidad de una ruptura superficial futura.

Los esfuerzos regionales del SFMA son transtensivos, donde el esfuerzo mínimo se localiza en dirección $\mathrm{NO}$ y el esfuerzo horizontal máximo $\left(\sigma_{1}\right.$ o $\sigma_{2}$ ) se dirige al NE, en correspondencia con la traza de las estructuras principales (Quintero-Legorreta, 2002; Garduño-Monroy et al., 2009; Pérez-Orozco et al., 2018). Esta orientación de esfuerzos ha prevalecido con algunas variaciones temporales desde el Mioceno Tardío. De acuerdo con Mennella et al. (2000), durante el Plioceno comenzó una fase transtensiva con el esfuerzo mínimo $\left(\sigma_{3}\right)$ orientado en dirección $\mathrm{NO}$, pero para el Pleistoceno Superior - Holoceno ocurrió una fase predominantemente extensiva, donde el esfuerzo mínimo rotó en sentido horario hasta una dirección NE.

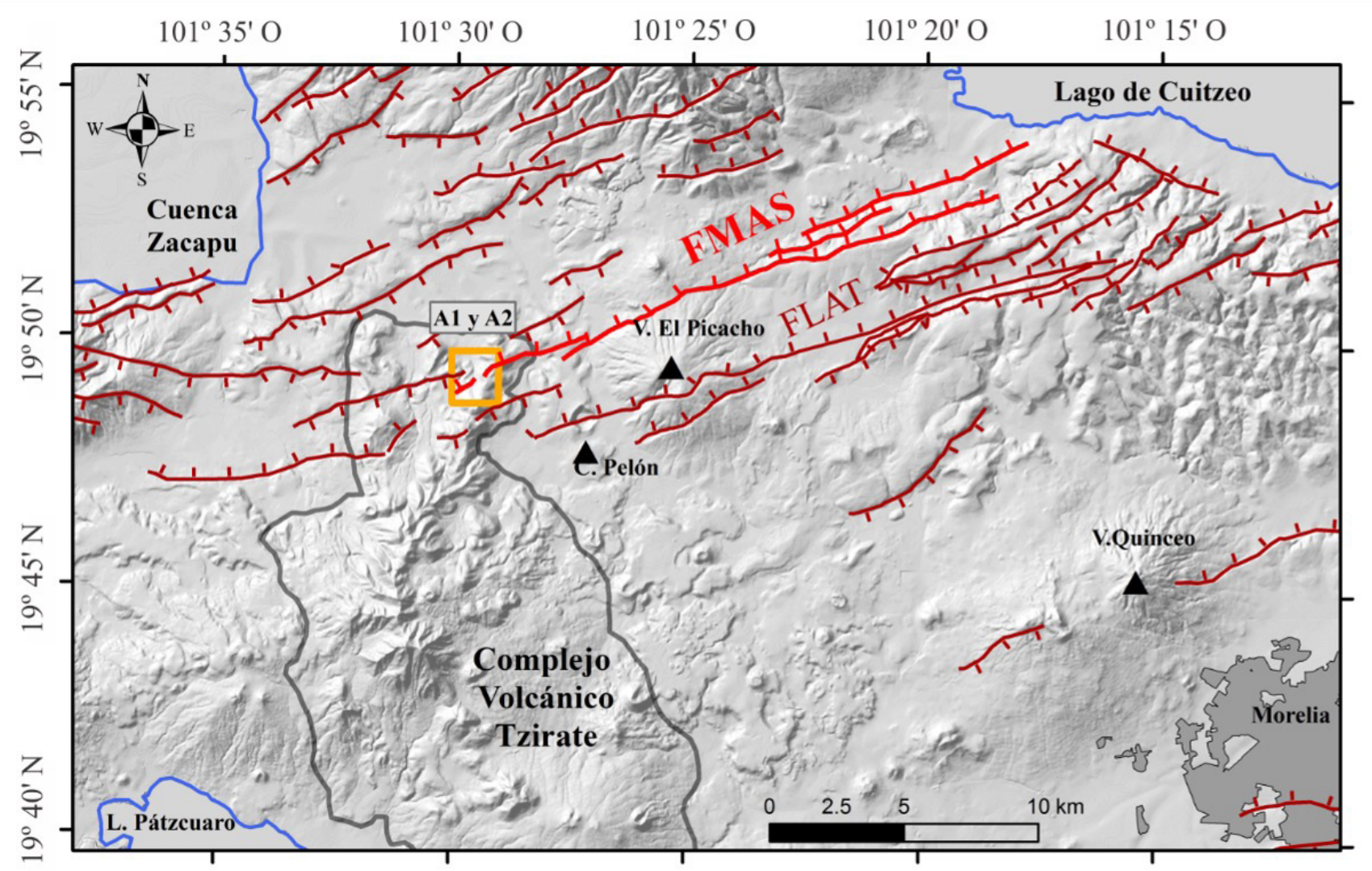

Figura 3 Ubicación de la falla El Malpaso-El Salto (FMAS), al norte de la ciudad de Morelia, en el estado de Michoacán. Se resaltan las seis trazas principales que la conforman y afectan las secuencias ignimbríticas del sur de Cuitzeo, las lavas de los volcanes El Picacho y Cerro Pelón y los depósitos del Complejo Volcánico El Tzirate (CVT). La figura también incluye la ubicación del afloramiento descrito en este trabajo (rectángulo naranja con la etiqueta A1 y A2). Abreviaturas: FLAT: falla La Alberca - Teremendo.Triángulos: volcanes principales. 
La región de estudio, ubicada al norte de Morelia (Figuras 3 y 4) está afectada por tres familias de fallas principales con direcciones NE, ENE y NO. De estas, la familia NO corresponde a estructuras más antiguas a la formación del SFMA (estructuras del basamento pre-Miocénico; Ego y Ansan, 2002; Garduño-Monroy et al., 2009), mientras que la familia ENE y la familia NE poseen evidencias cinemáticas que indican que se formaron en un campo de esfuerzos de transtensión lateral izquierda, donde el esfuerzo mínimo se orienta al $\mathrm{NO}\left(08^{\circ} / 147^{\circ} \mathrm{N}\right)$ y el esfuerzo máximo al NE $\left(27^{\circ} / 241^{\circ} \mathrm{N}\right)$, según reporta Pérez-Orozco et al., (2018, Figura 4).

De acuerdo con Ego y Ansan (2002), el desarrollo de estas estructuras se debe a los esfuerzos generados por la subducción oblicua en la zona.

La influencia de la deformación transtensiva y las rotaciones locales del campo de esfuerzos se expresan en el paisaje mediante el desarrollo notable de geometrías en échelon, estructuras de bajo ángulo, alto ángulo, de tensión, estructuras en flor, terminaciones de cola de caballo, así como pilares y cuencas tipo pull-apart; este tipo de geometrías han sido previamente reportadas para otras partes del CVTM por Martínez-Reyes y Nieto-Samaniego (1990), García-Palomo et al. (2009), Suter et al. (2001), Garduño-Monroy et al. (2009) y Mennella et al. (2000, 2011).

Las fallas con orientación ENE y algunas NE presentan un componente principal normal con una ligera componente de desplazamiento lateral izquierda, debido a la extensión en sentido NO (Mennella, 2011). En el caso de las fallas con orientación NO y NE, parecen ser reactivadas por el campo de esfuerzos regional actual, produciendo, en su mayoría, fallamiento de tipo normal. Sin embargo, algunas muestran además

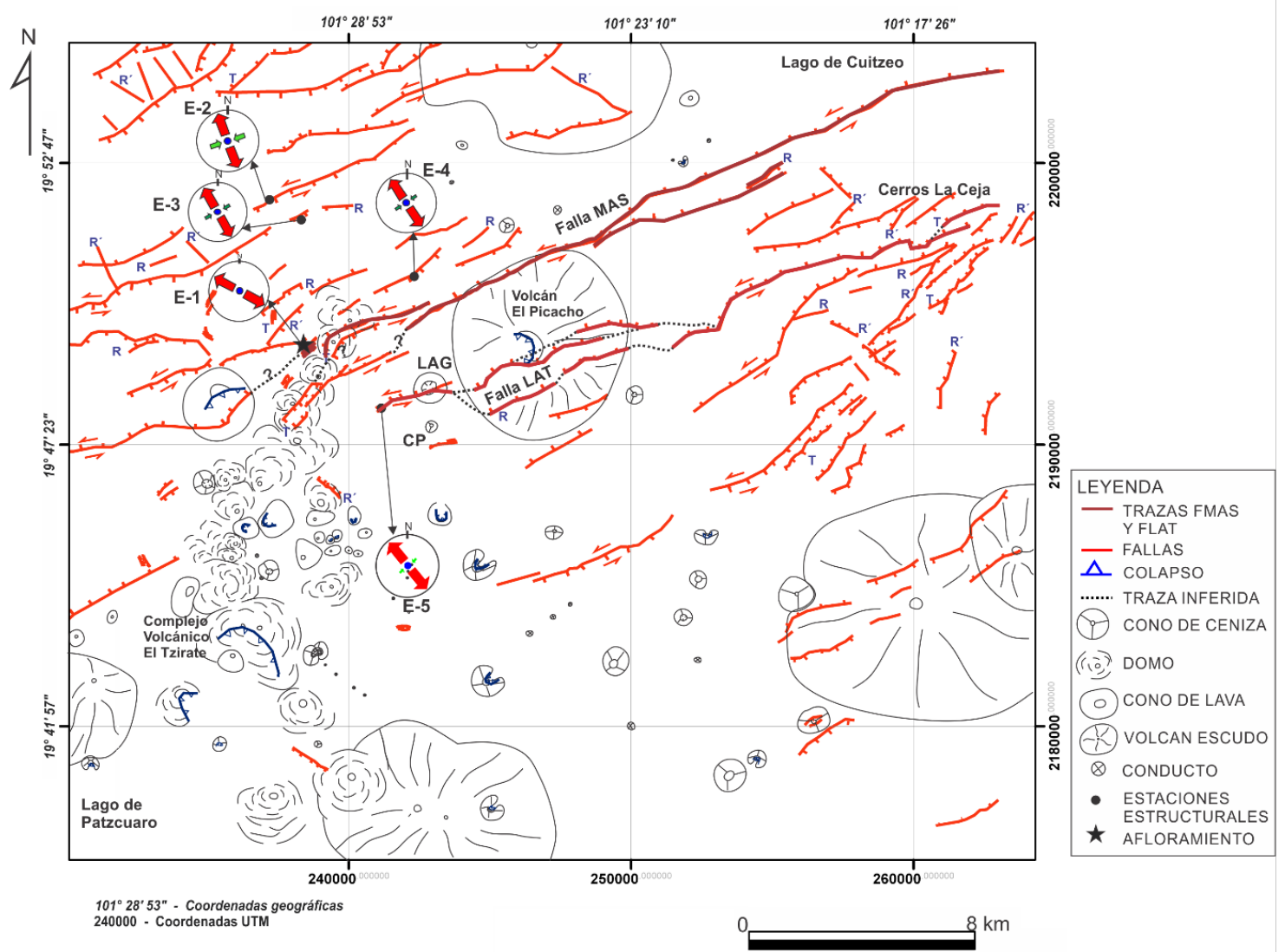

Figura 4 Mapa estructural de la región de estudio resaltando las trazas de las fallas El Malpaso-El Salto y La Alberca-Teremendo, así como las estructuras de alto y bajo ángulo (estructuras de relevo). Las fallas indican el campo de esfuerzos actual en la región (Modificada de Pérez-Orozco et al., 2018). 
una componente de desplazamiento lateral, ya sea izquierdo para la familia NE o derecho para la NO (Ego y Ansan, 2002; Mennella, 2011).

En la zona de estudio destacan los depósitos félsicos derivados de la actividad del Complejo Volcánico El Tzirate (CVT), los cuales se disponen en forma de $\mathrm{C}$ abierta, con la porción sur alineada en sentido NNO y los depósitos del norte alineados en sentido NNE (Figuras 3 y 4), probablemente respondiendo a una estructura del basamento en la misma dirección (Pérez-Orozco et al., 2018); esta orientación concuerda con la fase transtensiva del Plioceno (Mennella et al., 2000).

En la ladera este del CVT se observa la alineación de seis centros monogenéticos de composición andesítica del Pleistoceno Tardío - Holoceno, cuya orientación concuerda con la fase extensiva sugerida por Mennella et al. (2000).

De acuerdo con Pérez-Orozco et al. (2018), la formación del CVT está condicionada por la transtensión y la variación temporal del campo de esfuerzos, donde el magmatismo félsico se ubica en las zonas de relevo en échelon orientadas al NNE. Actualmente, el CVT representa un alto estructural a cuyos lados se observan fallas normales y la formación de grábenes de tamaños variables entre $\sim 4 \mathrm{~km}$ (zona Malpaso) y $\sim 12 \mathrm{~km}$ de longitud (zonas Zacapu y Cuitzeo).

\section{Métodos}

En este trabajo se realizaron análisis morfológicos y estructurales convencionales para identificar estructuras relacionadas a la actividad sísmica, evaluar la influencia de las fallas tectónicamente activas en el paisaje y localizar sitios de afloramiento adecuados para el análisis paleosísmico. Los insumos cartográficos utilizados fueron obtenidos del Instituto Nacional de Estadística y Geografía (INEGI, 2010) y consisten en mapas topográficos con escala 1: 50 000, fotografías aéreas con escala 1:20 000 e imágenes LIDAR con resolución de 15 metros; estos insumos fueron trabajados en varias plataformas de SIG (ArcMap y Global Mapper). Con estos insumos se realizó el análisis morfoestructural de las trazas de falla de la zona. Las medidas de longitud de las trazas de fallas y la altura de los escarpes acumulados fueron obtenidas a través de la repetición de mediciones y perfiles topográficos respectivamente, obtenidos en ambas plataformas SIG (10 mediciones en cada una).

El análisis paleosísmico se enfocó en el segmento occidental de la FMAS, donde el escarpe esta suavizado en la superficie y en el área de influencia de las estructuras de relevo (NNE-SSO), los planos de estas estructuras se observan expuestos en un afloramiento de $100 \mathrm{~m}$ de largo a pie de carretera. El análisis de los materiales aflorantes siguió las guías metodológicas detalladas por McCalpin (1996), con la creación de un fotolog de trabajo ajustado con puntos de referencia ubicados en la cima y la base del afloramiento, el cual permitió identificar las unidades litológicas guías o marcadoras sobre las que se midieron los desplazamientos individuales asociados a cada plano de falla expuesto.

Las mediciones de estos desplazamientos individuales mostraron errores asociados en el rango de $\pm 1 \mathrm{~cm}$ (fallas f3a, f5a y F8, Tabla 1) a $\pm 14 \mathrm{~cm}$ (falla F4), con un promedio de error de $\pm 7 \mathrm{~cm}$, esto debido a las irregularidades en los límites de las unidades litológicas guías; a este error promedio se suma $\pm 2 \mathrm{~cm}$ que correspondería al error metodológico durante la digitalización del log, lo cual nos genera un error general promedio de $\pm 9 \mathrm{~cm}$ para las mediciones de desplazamientos individuales de las fallas expuestas en el afloramiento. Finalmente, se construyó una columna tipo para la secuencia expuesta.

Los parámetros de actividad de la falla que se resolvieron para este trabajo fueron: tasa de desplazamiento, período de recurrencia de la falla y magnitud máxima esperada para un evento futuro de ruptura de la falla.

Para estimar la tasa de desplazamiento (SR por sus siglas en inglés) se utilizó la cantidad de movimiento vertical acumulado en la traza de falla principal y el tiempo transcurrido desde el primer evento registrado en el afloramiento (que en este caso corresponde a la edad de las unidades estratigráficas afectadas), considerando la relación propuesta por McCalpin (1996): 
Tabla 1. Resumen de las características de las fallas observadas en el afloramiento, incluyendo los desplazamientos verticales medidos. *Desplazamiento total medido para la falla sumando el plano principal y los secundarios (p.e. F5 + f5a + f5b = desplazamiento vertical total en F5).

\begin{tabular}{|c|c|c|c|c|c|c|c|}
\hline $\begin{array}{l}\text { Sección del } \\
\text { afloramiento } \\
\text { (dirección) }\end{array}$ & Falla & Dirección & Echado & \multicolumn{2}{|c|}{$\begin{array}{l}\text { Desplazamien } \\
\text { to vertical } \\
(\mathrm{cm})\end{array}$} & $\begin{array}{l}\text { Unidad en } \\
\text { que se midió } \\
\text { el DV }\end{array}$ & $\begin{array}{l}\text { Apertura } \\
\text { horizontal } \\
(\mathrm{cm})\end{array}$ \\
\hline \multirow[t]{8}{*}{$\mathrm{A} 1(\mathrm{E}-\mathrm{O})$} & $\mathrm{F} 1$ & 233 & $86 \mathrm{NO}$ & \multicolumn{2}{|c|}{$32 \pm 9$} & \multirow{8}{*}{$\begin{array}{l}\text { Unidad 3: } \\
\text { FBC }\end{array}$} & 37 \\
\hline & F2 & 046 & $78 \mathrm{SE}$ & $\begin{array}{l}37 \pm \\
4\end{array}$ & \multirow[t]{2}{*}{$\begin{array}{l}45 \pm \\
7^{*}\end{array}$} & & 5 \\
\hline & $\mathrm{f} 2 \mathrm{a}$ & 023 & $72 \mathrm{SE}$ & $8 \pm 3$ & & & 3 \\
\hline & F3 & 245 & $83 \mathrm{NO}$ & $\begin{array}{l}11 \pm \\
4\end{array}$ & \multirow[t]{2}{*}{$\begin{array}{l}14 \pm \\
5^{*}\end{array}$} & & 5 \\
\hline & f3a & & & $3 \pm 1$ & & & 4 \\
\hline & F7 & 188 & $78 \mathrm{O}$ & \multicolumn{2}{|c|}{$18 \pm 6$} & & No \\
\hline & F8 & 221 & $82 \mathrm{NO}$ & \multicolumn{2}{|l|}{$3 \pm 1$} & & \\
\hline & F9 & 015 & $80 \mathrm{E}$ & \multicolumn{2}{|c|}{$22 \pm 8$} & & \\
\hline \multirow[t]{4}{*}{$\mathrm{A} 2(\mathrm{~N}-\mathrm{S})$} & $\mathrm{F} 4$ & 240 & $56 \mathrm{NO}$ & \multicolumn{2}{|c|}{$84 \pm 14$} & Unidad 2: & 30 \\
\hline & F5 & 252 & $89 \mathrm{NO}$ & $\begin{array}{l}10 \pm \\
3\end{array}$ & \multirow[t]{3}{*}{$\begin{array}{l}24 \pm \\
8^{*}\end{array}$} & \multirow[t]{3}{*}{$\begin{array}{l}\text { Unidad 2: } \\
\text { DCC }\end{array}$} & 4 \\
\hline & f5a & 250 & $75 \mathrm{NO}$ & $8 \pm 4$ & & & 2 \\
\hline & $\mathrm{f} 5 \mathrm{~b}$ & 245 & $71 \mathrm{NO}$ & $6 \pm 1$ & & & 1 \\
\hline
\end{tabular}

$S R=$ Desplazamiento total $(\mathrm{mm}) /$ Tiempo transcurrido (años) 1)

Para estimar los períodos o intervalos de recurrencia (RI por sus siglas en inglés) se puede usar el método directo, que produce una recurrencia media, o bien el método geológico, que ayuda a caracterizar los ciclos sísmicos. En este trabajo, por las limitaciones del análisis, el intervalo de recurrencia se calculó usando el método directo, mediante la relación (McCalpin, 1996):

$R I=$ Dvprom $/(\mathrm{SR}-\mathrm{C})$

Donde: Dvprom es el desplazamiento vertical promedio (en mm); SR es la tasa de desplazamiento cosísmico (en mm/año); y C es la tasa de desplazamiento producido por creep.

Finalmente, la magnitud probable para la ruptura de una falla puede ser estimada usando relaciones empíricas que usan parámetros conocidos de la falla, tales como: la longitud de ruptura superficial, el desplazamiento vertical por evento, la tasa de desplazamiento y el período de recurrencia. Las relaciones empíricas más usadas utilizan la longitud de ruptura superficial (LRS), pero su principal incertidumbre radica en la falta de información acerca de la geometría y la longitud prehistóricas de las fallas en estudio (Lafuente, $2011)$. Para este trabajo, se utilizaron cuatro relaciones empíricas que usan LRS con la finalidad de comparar las magnitudes estimadas con cada 
aproximación y con los trabajos publicados para otras fallas del SFMA. Las relaciones usadas en este trabajo son las ecuaciones de Wells y Coppersmith (1994), Anderson et al. (1996), Stirling et al. (2002) y Wesnousky (2008; Tabla 1).

\section{Análisis morfoestructural de la falla El Malpaso - El Salto}

La falla El Malpaso - El Salto (FMAS) muestra un escarpe prominente tanto en los mapas topográficos, como en las fotos aéreas y el LIDAR, siendo este último el preferido para realizar el análisis morfoestructural de las trazas de la falla. Esta falla posee una dirección promedio de $\mathrm{N} 250^{\circ}$, con una inclinación de $56^{\circ}-75^{\circ}$ hacia el $\mathrm{NO}$ y escarpes con saltos acumulados que varían desde un mínimo de $4 \mathrm{~m}$ hasta máximos de $120 \mathrm{~m}$. La falla está formada por seis trazas con direcciones $\mathrm{N} 243-265^{\circ}$ y con un arreglo en échelon en donde destacan una traza principal o maestra de $\sim 19 \mathrm{~km}$ y cinco trazas secundarias con longitudes entre 3 y $7 \mathrm{~km}$. Estas trazas se observan separadas entre sí por distancias menores a $1 \mathrm{~km}$ (Figuras 3 y 4 ).

Hacia el extremo suroeste de las trazas secundarias se observan dos trazas de longitudes $\sim 2 \mathrm{~km}$, separadas por una distancia $\leq 3 \mathrm{~km}$, estas podrían estar relacionadas al área de influencia subsuperficial de la FMAS, pero no fueron incluidas en este análisis debido a la distancia de separación, a la falta de datos geofísicos que confirmen su asociación con la traza principal y a que se decidió evaluar primero las trazas más continuas posibles. La longitud total de las trazas combinadas alcanza $28 \mathrm{~km}$ en superficie (Figuras 3 y 4). Esta falla ocasiona el basculamiento del bloque de techo un mínimo de $3^{\circ}$ (medido en los perfiles topográficos en el sector oeste de la falla, perpendiculares a la traza) y un máximo de $12^{\circ}$ (medido en el afloramiento descrito en este trabajo) hacia el noroeste. Para las fallas de esta región, algunos autores han propuesto que sus planos presentan una geometría lístrica, con una inclinación promedio de $60^{\circ}$, los cuales generan el basculamiento de los bloques hacia el sur apreciable en el paisaje (p.e. bloque
Morelia), estas características sugieren la probabilidad de que exista en la región una conexión subsuperficial entre trazas de falla vecinas cercanas (Israde-Alcántara y Garduño-Monroy, 1999; Garduño-Monroy et al., 2009; Israde-Alcántara et al., 2010; Gómez-Vasconcelos et al., 2020).

La FMAS exhibe características de movimiento de tipo normal con el bloque de techo cayendo hacia el nor-noroeste, combinado con una ligera componente de desplazamiento lateral izquierdo (reportada también por Pérez-Orozco et al., 2018), lo que provoca el desvío de las corrientes de drenaje en la misma dirección (observable particularmente en la traza principal que corta al volcán El Picacho) y podría relacionarse con el alargamiento de los depósitos ignimbríticos de Copándaro (Cerros La Ceja), observable al sur del Lago de Cuitzeo (Figuras 3 y 4).

De este a oeste, las primeras trazas dislocan, levantan, rotan y basculan bloques de las secuencias piroclásticas de los Cerros La Ceja, cuyas unidades pertenecen a la ignimbrita Cuitzeo de una edad de 18.5 a 17.4 Ma (Avellán et al., 2020) y afecta las secuencias fluvio-lacustres pertenecientes al lago de Cuitzeo, también de edad miocénica, formando escarpes pronunciados y de hasta 120 m de altura (Figura 5). El segmento central corta la ladera norte del volcán tipo escudo El Picacho y el flujo de lavas del cono El Pelón, ambos con una edad de $1.5 \pm 0.12 \mathrm{Ma}$ (Kshirsagar et al., 2015), aquí el escarpe se suaviza y alcanza alturas de 50 a $70 \mathrm{~m}$. Por último, los segmentos occidentales cortan y desplazan los depósitos más septentrionales del Complejo Volcánico El Tzirate, de edad de 1.4 $\pm 0.07 \mathrm{Ma}$ (Kshirsagar et al., 2015), formando estructuras de pilares y cuencas pull-apart (Figura 4), en esta zona el escarpe alcanza alturas máximas de 4 m y va suavizándose cada vez más hasta desaparecer.

Para esta falla, Suter et al. (2001) calcularon una tasa de desplazamiento vertical de $0.16 \mathrm{~mm} / \mathrm{año}$, usando la expresión geomorfológica de escarpes pronunciados (multi-evento) con alturas mayores a $100 \mathrm{~m}$, los cuales cortan unidades basálticas pleistocénicas (0.87-0.75 Ma) e ignimbritas del Plioceno Tardío, ambas unidades del Lago de 
Cuitzeo; estos escarpes también afectan andesitas y basaltos del Mioceno Tardío de Tarímbaro. Cabe mencionar que estos autores incluyen esta falla en la zona de fallas Zacapu-Salvatierra, pero indican características similares a las de las fallas de Morelia que se agrupan dentro del SFMA.

\section{Análisis paleosismológico}

\subsection{DESGRIPGIÓN DEL AFLORAMIENTO}

El afloramiento descrito en este trabajo es un corte artificial expuesto al costado de la carretera rural que va de los poblados Teremendo de los Reyes a Tacupillo - Huaniqueo (19 49'08.24'N, $101^{\circ}$ $30^{\prime} 23.3$ ”'O, a 2033 msnm; Figuras 3 y 5) y la cual presenta una dirección E-O. Se ubica en la ladera noroeste del CVT, sobre lomeríos compuestos por depósitos piroclásticos localizados al oeste del domo La Cruz.

En este punto, morfológicamente el escarpe es poco visible y el afloramiento se localiza en un pequeño puerto interlomas donde la pendiente es mínima $\left(<5^{\circ}\right)$ en comparación con las zonas adyacentes que presentan pendientes más pronunciadas $\left(15-20^{\circ}\right)$.

El corte es recto en su porción sur y posee forma de "L" en su porción norte, lo cual permite la exposición de paredes con orientación este - oeste $\left(\mathrm{N} 085^{\circ}\right)$ y con orientación norte - sur. La porción norte del afloramiento posee dimensiones de $\sim 100$ $\mathrm{m}$ de longitud (pared con dirección este-oeste), $\sim 50 \mathrm{~m}$ de longitud (pared con dirección norte-sur) y una altura promedio de $4 \mathrm{~m}$ (Figura 5).

La base del afloramiento se encuentra a nivel de la carretera y su cima en terrenos ejidales que muestran evidencias de actividad humana (p.e. cercados, emparejamiento del terreno). Es un afloramiento heterolitológico compuesto por flujos piroclásticos, con tendencia intermedia a félsica, en los cuales se observa como fases minerales más abundantes al cuarzo y la biotita, así como abundantes cantidades de pómez.

Toda la secuencia es afectada por fallamiento normal distribuido en tres familias, la más
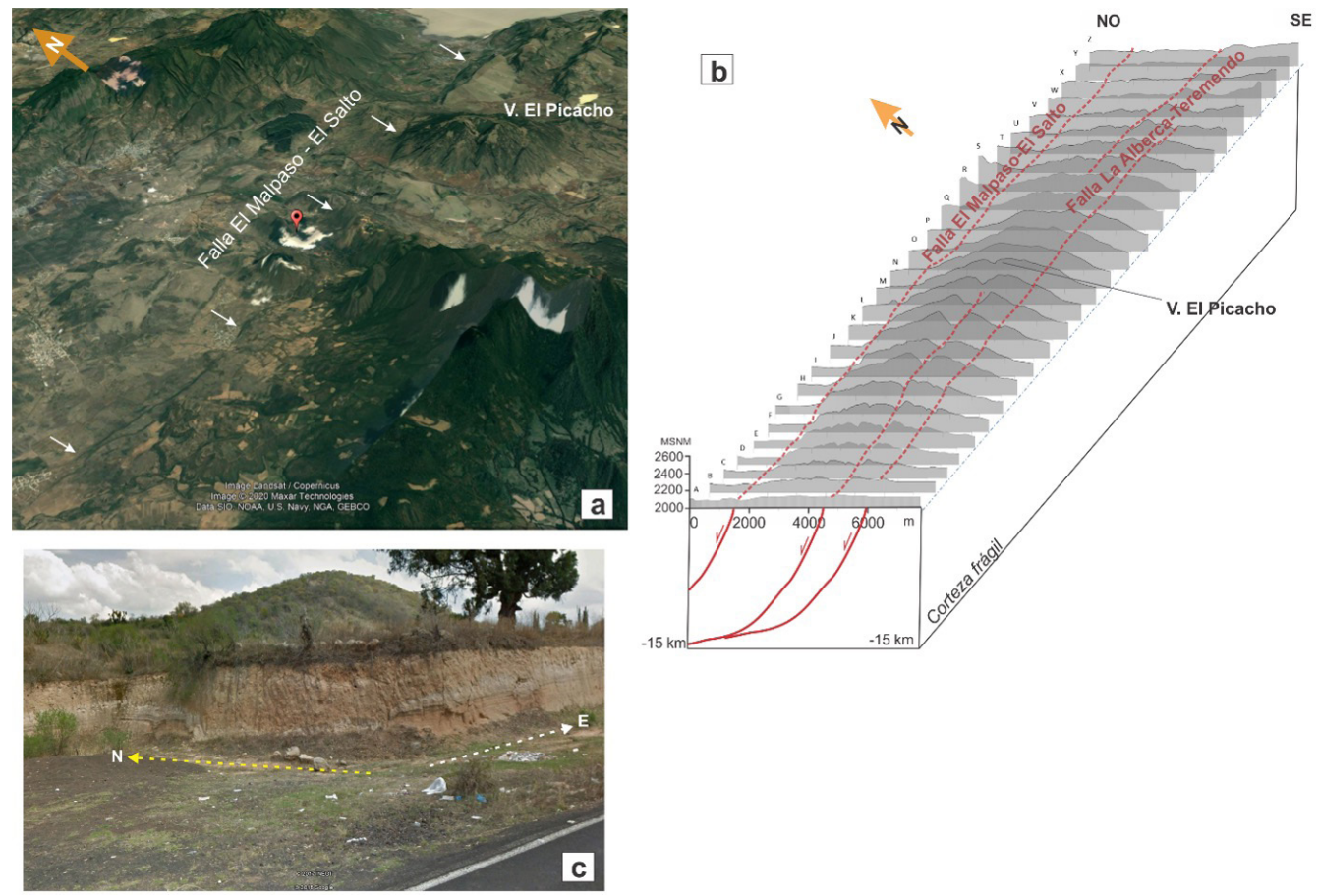

Figura 5 a) Vista aérea de la Falla El Malpaso - El Salto observada desde el poniente. El marcador rojo indica la localización del afloramiento. Imagen obtenida con el software Google Earth Pro. b) Perfiles realizados sobre la traza de la Falla El Malpaso - El Salto afectando al volcán El Picacho, así como la traza de la Falla La Alberca-Teremendo al sur (coordenadas de los perfiles en la Tabla complementaria 1). c) Vista del afloramiento analizado. 
abundante presenta orientaciones NE y las menos frecuentes poseen orientaciones ENE y N - S.

En este afloramiento se observa la traza de la falla regional El Malpaso-El Salto (FMAS).

La estratigrafía del afloramiento se compone de 4 unidades principales, diferenciadas por sus atributos de granulometría, tipos de componentes y estilo de depósito (Figura 6).

De la base a la cima la secuencia está conformada por:
UNIDAD 1 - FHc1: Depósito ignimbrítico con un espesor visible de $175 \mathrm{~cm}$, de color café claro a anaranjado, de composición heterolitológica, con componentes félsicos, posee fragmentos líticos sub-redondeados de tendencia dacítica y riolítica, así como elevados contenidos de biotitas, cuarzo y pómez. Esta unidad fue clasificada como un flujo hiperconcentrado (FHcl) y está afectada por fallas normales aunque la deformación total no pudo ser cuantificada.
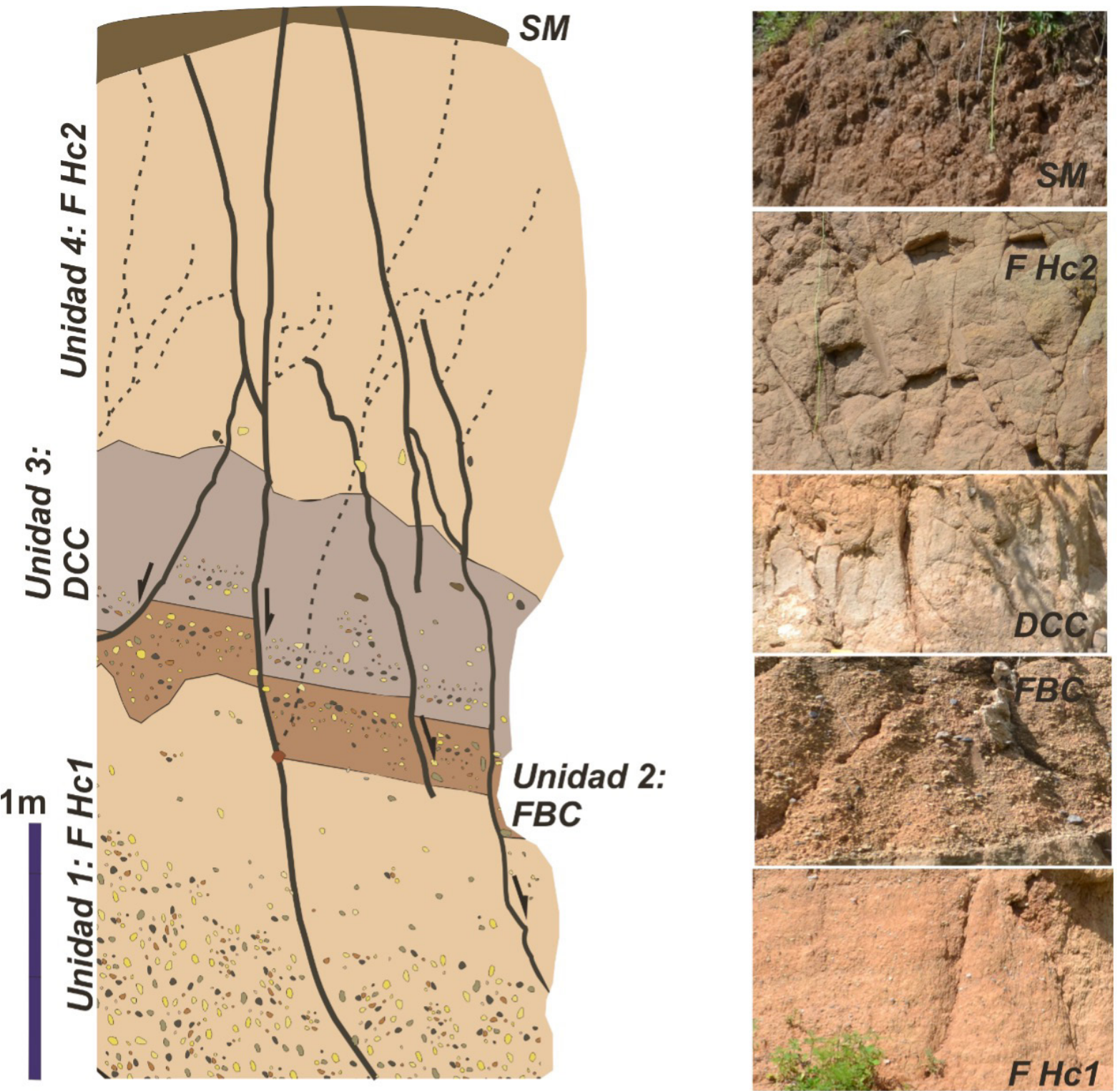

Figura 6 Columna estratigráfica simplificada de los materiales expuestos en el afloramiento, identificación de litofacies y correspondiente registro fotográfico. Abreviaturas: SM: Suelo moderno; FHc2: Flujo hiperconcentrado más reciente (Unidad 4); DCC: Depósito de caída de cenizas (Unidad 3); FBC: Flujo de bloques y cenizas (Unidad 2); FHc1: Flujo hiperconcentrado basal (Unidad 1). 
UNIDAD 2 - FBC: Depósito de un flujo de bloques y ceniza $(\mathrm{FBC})$ con un espesor promedio de $15 \mathrm{~cm}$, color café claro y con una estructura de gradación normal en su depósito. Su composición es dacítica con algunos fragmentos esporádicos sub-angulosos de andesitas con diámetros máximos de $5 \mathrm{~cm}$. Esta unidad está afectada por las fallas principales y secundarias del afloramiento y en esta se cuantificó la mayoría de los desplazamientos verticales aquí reportados.

UNIDAD 3 - DCG: Depósito de cenizas color gris claro y geometría acuñada, presentando espesores mayores en la sección norte del afloramiento (hasta $50 \mathrm{~cm}$ ) y menores en la sección este $(20-25 \mathrm{~cm}$ hasta desaparecer), lo cual refleja el basculamiento de los depósitos. Su composición es predominantemente dacítica con abundancia de componentes de tendencia félsica. La unidad se identificó como un depósito de caída de ceniza (DCG) y está desplazada en la vertical un mínimo de $17 \mathrm{~cm}$ y un máximo de $84 \mathrm{~cm}$ por fallamiento normal.

UNIDAD 4 - FHc2: Depósito ignimbrítico de -1 m de espesor, color café claro a anaranjado, con composición heterolitológica, pero predominantemente félsica, presenta fragmentos líticos sub-redondeados de afinidad dacítica y riolítica, así como abundantes cantidades de fragmentos de pómez, cristales de biotita y cuarzo.

Esta unidad fue clasificada como un flujo hiperconcentrado con alta porosidad (FHc2) y está afectado por fallamiento normal.

La deformación solo pudo ser cuantificada en su contacto con la unidad 2 (desplazamiento vertical $\sim 25 \mathrm{~cm}$ ), sin embargo, hacia la superficie del terreno no se observa ningún escarpe. Cubriendo toda la secuencia, y de manera directa a la unidad 4 , se encuentra el suelo moderno (SM) en desarrollo, con un espesor máximo de $20 \mathrm{~cm}$, el cual no está afectado visiblemente por el fallamiento.

Esta secuencia estratigráfica fue asociada a una edad de $1.4 \pm 0.07$ Ma de acuerdo con Kshirsagar et al. (2015).

\subsection{ESTRUCTURAS IDENTIFICADAS EN EL AFLORAMIENTO}

Como se mencionó previamente, toda la secuencia estratigráfica expuesta es afectada por fallamiento normal, con estructuras conjugadas y fracturamiento.
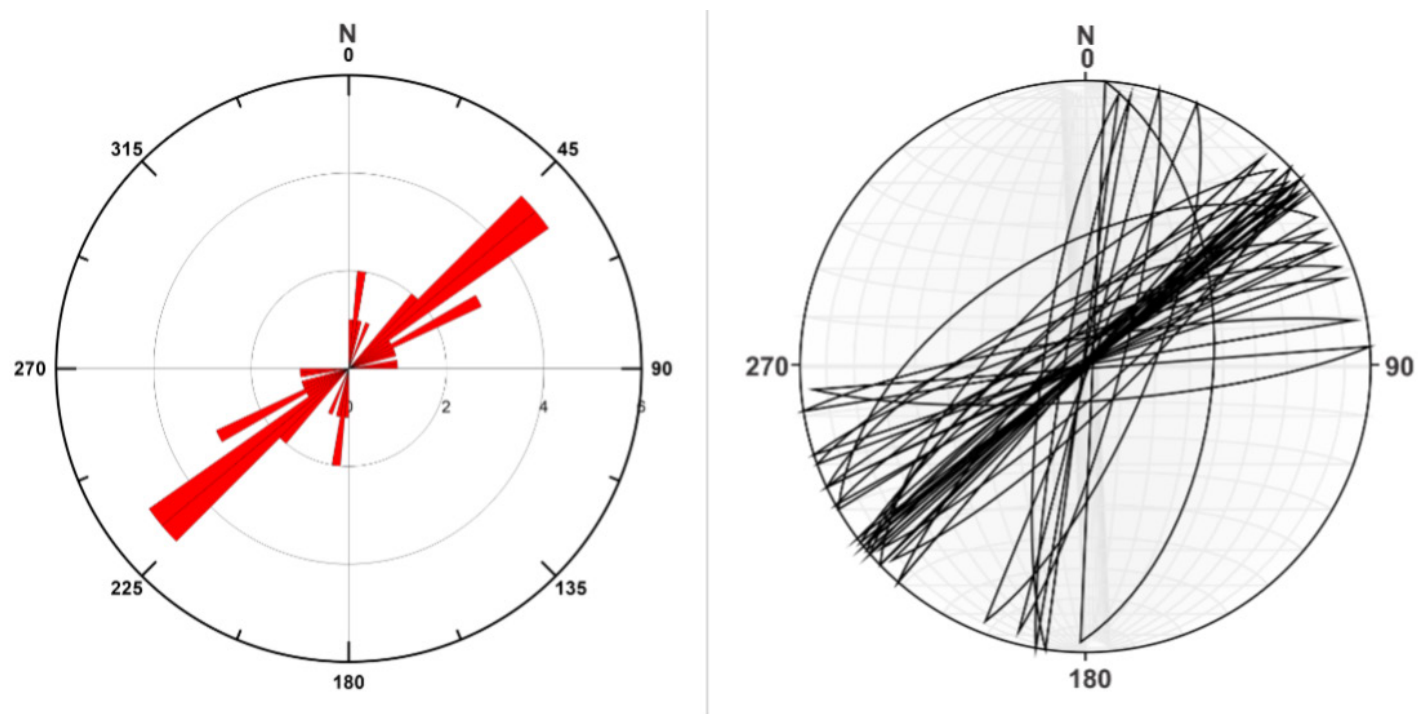

Figura 7 Orientaciones de las familias de estructuras principales medidas en el afloramiento A1, al norte del Complejo Volcánico El Tzirate (CVT). 
Tabla 2. Resumen de las características de las fallas observadas en el afloramiento, incluyendo los desplazamientos verticales medidos. *Desplazamiento total medido para la falla sumando el plano principal y los secundarios (p.e. F5 + f5a + f5b = desplazamiento vertical total en F5).

\begin{tabular}{|c|c|c|c|c|c|c|c|}
\hline $\begin{array}{l}\text { Sección del afloramiento } \\
\text { (dirección) }\end{array}$ & Falla & Dirección & Echado & \multicolumn{2}{|c|}{$\begin{array}{l}\text { Desplazamiento vertical } \\
(\mathrm{cm})\end{array}$} & $\begin{array}{l}\text { Unidad en que se midió el } \\
\text { DV }\end{array}$ & $\begin{array}{l}\text { Apertura horizontal } \\
(\mathrm{cm})\end{array}$ \\
\hline \multirow[t]{8}{*}{$\mathrm{A} 1(\mathrm{E}-\mathrm{O})$} & $\mathrm{F} 1$ & 233 & $86 \mathrm{NO}$ & \multicolumn{2}{|l|}{$32 \pm 9$} & Unidad 3: FBC & 37 \\
\hline & $\mathrm{F} 2$ & 046 & $78 \mathrm{SE}$ & $37 \pm 4$ & \multirow[t]{2}{*}{$45 \pm 7^{*}$} & & 5 \\
\hline & $\mathrm{f} 2 \mathrm{a}$ & 023 & $72 \mathrm{SE}$ & $8 \pm 3$ & & & 3 \\
\hline & F3 & \multirow[t]{2}{*}{245} & \multirow[t]{2}{*}{$83 \mathrm{NO}$} & $11 \pm 4$ & \multirow[t]{2}{*}{$14 \pm 5^{*}$} & & 5 \\
\hline & f3a & & & $3 \pm 1$ & & & 4 \\
\hline & F7 & 188 & $78 \mathrm{O}$ & \multicolumn{2}{|l|}{$18 \pm 6$} & & \multirow[t]{3}{*}{ No observado } \\
\hline & F8 & 221 & $82 \mathrm{NO}$ & \multicolumn{2}{|l|}{$3 \pm 1$} & & \\
\hline & F9 & 015 & $80 \mathrm{E}$ & \multicolumn{2}{|l|}{$22 \pm 8$} & & \\
\hline \multirow[t]{6}{*}{$\mathrm{A} 2(\mathrm{~N}-\mathrm{S})$} & F4 & 240 & $56 \mathrm{NO}$ & \multicolumn{2}{|c|}{$84 \pm 14$} & Unidad 2: DCC & 30 \\
\hline & F5 & 252 & $89 \mathrm{NO}$ & $10 \pm 3$ & \multirow[t]{3}{*}{$24 \pm 8^{*}$} & \multirow[t]{3}{*}{ Unidad 2: DCC } & 4 \\
\hline & f5a & 250 & $75 \mathrm{NO}$ & $8 \pm 4$ & & & 2 \\
\hline & $\mathrm{f} 5 \mathrm{~b}$ & 245 & $71 \mathrm{NO}$ & $6 \pm 1$ & & & 1 \\
\hline & F6 & \multirow[t]{2}{*}{046} & \multirow[t]{2}{*}{$88 \mathrm{SE}$} & $13 \pm 4$ & \multirow[t]{2}{*}{$22 \pm 7^{*}$} & \multirow[t]{2}{*}{ Unidad 3: FBC } & \multirow[t]{2}{*}{ No observado } \\
\hline & f6a & & & $9 \pm 3$ & & & \\
\hline
\end{tabular}

Estas estructuras frecuentemente son rellenadas por materiales secundarios, que pueden ser mezclas de las unidades litológicas de la cima, harina de falla, así como pómez fibrosa color gris amarillento que contiene cristales de plagioclasas, biotitas y anfíboles, así como algunos fragmentos líticos sub-angulosos de composición andesítica y diámetro variable (que va desde algunos milímetros hasta $1 \mathrm{~cm}$ ), este último tipo de relleno aparece preferentemente en las estructuras NE (Pérez-Orozco et al., 2018).

Las fallas y fracturas observadas se agrupan en una gran familia con dirección preferencial NE, $\mathrm{y}$ dos familias menos abundantes de estructuras con dirección N-S y ENE (Figura 7). En algunas porciones del afloramiento, las fallas más grandes presentan planos bien definidos que afectan toda la secuencia y provocan desplazamientos verticales y aberturas en sentido horizontal de hasta $37 \mathrm{~cm}$ (mínimo $2 \mathrm{~cm}$ ).
Mientras tanto, en otros puntos del afloramiento, el desplazamiento total acumulado es repartido en una falla principal con menor desplazamiento y varias fallas secundarias asociadas, paralelas en sentido y desplazamiento a la falla principal. Debido a las dimensiones y variación en la orientación del afloramiento, el análisis de la deformación fue separado en dos secciones: sección Al, la cual corresponde a la pared con orientación este - oeste y sección A2, la cual corresponde a la pared con orientación norte - sur. Las características más importantes descritas para las fallas de las secciones siguientes se encuentran resumidas en la Tabla 2.

\subsubsection{DEFORMACIÓN EN LA SECGIÓN A1}

En esta pared se observan tres planos de falla principales, las cuales cortan toda la secuencia hasta la superficie. Sin embargo, una vez que la 
alcanzan, no generan ningún escarpe visible. Esto puede ser producto de la actividad antropogénica para nivelar la superficie del terreno, o bien puede deberse a un desplazamiento superficial muy pequeño que forma un escalón dificil de identificar y fue rápidamente erosionado. De este a oeste, el afloramiento presenta las fallas principales F1, F2 y F3, y las fallas secundarias F7, F8 y F9. Todas las fallas observadas muestran un comportamiento de fracturas hacia la superficie, rellenándose de los materiales de las unidades litológicas superiores o bien de pómez fibrosa, pero sin que se observe una cuña coluvial clara asociada, por lo cual son clasificadas como fallas mixtas.

La falla principal (F1, en la Figura 8) corresponde a una falla de movimiento normal con el bloque de techo cayendo al noroeste. Posee una orientación de $\mathrm{N} 233^{\circ}$ e inclinación de $86^{\circ} \mathrm{NO}$. Esta falla se rellena del material de las unidades 2 y 4 (F Hc2 y FBC), aquí la unidad 3 (DCG) no está presente.
El desplazamiento vertical total registrado para esta falla fue de $32 \pm 9 \mathrm{~cm}$ (medido en la unidad 3), ocasionando la rotación del bloque de techo que genera una apertura horizontal máxima de $37 \mathrm{~cm}$. Asociadas a esta falla se encuentran 12 fracturas de tamaño variable que aparentemente no desplazan verticalmente las capas de la secuencia, pero algunas de ellas presentan aperturas horizontales menores a $5 \mathrm{~cm}$.

La segunda falla en importancia (F2, en la Figura 9) presenta movimiento normal, con el bloque de techo cayendo al sureste. Corta toda la secuencia hasta el suelo actual pero tampoco se observa un escarpe en superficie. Posee una orientación de $\mathrm{N} 046^{\circ}$ y una inclinación de $78^{\circ} \mathrm{SE}$. El desplazamiento vertical total de las capas es de $37 \pm 4 \mathrm{~cm}$, notorios en la unidad estratigráfica 3. Su apertura horizontal máxima es de $5 \mathrm{~cm}$ y se rellena del material de la unidad 4 y pómez fibrosa (Figura 9). Asociada a F2 se presenta una falla secundaria de bajo ángulo (f2a) que tiene una
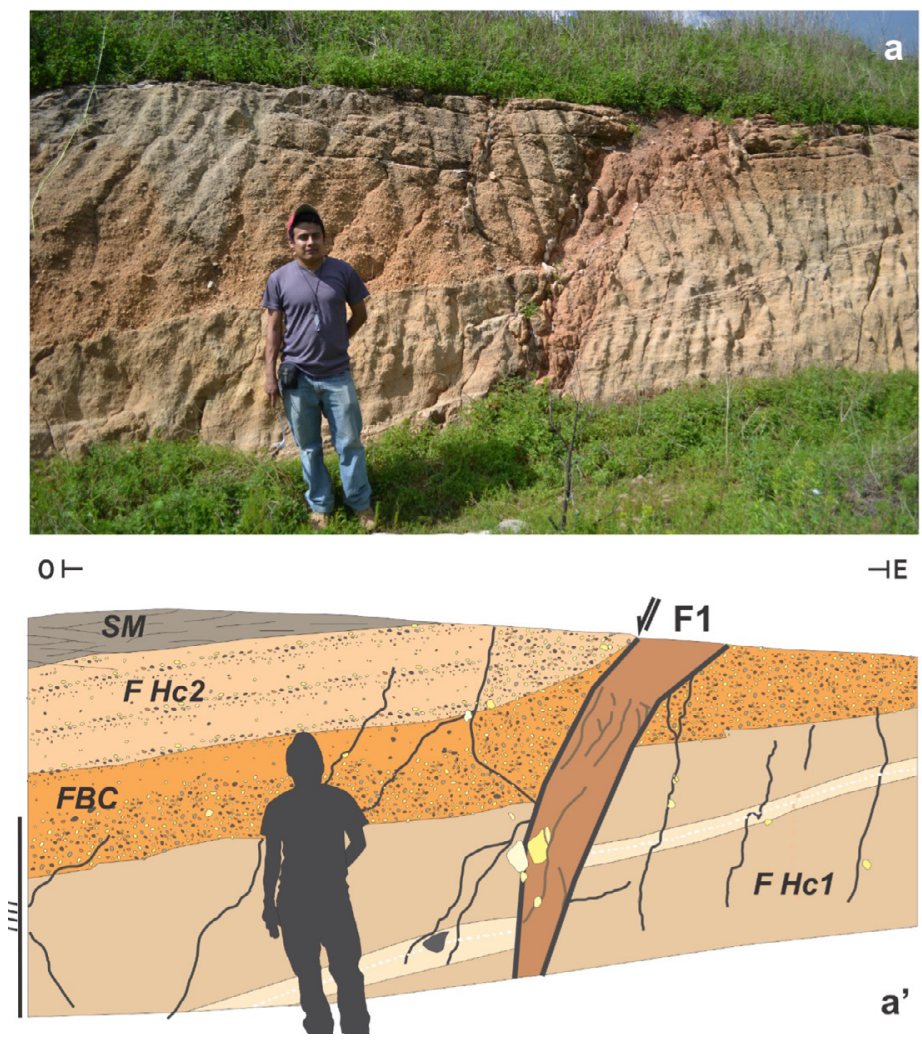

Figura 8 a) Fotografía e interpretación (a') de la estructura F1 en el afloramiento A1. Escala humana $1.6 \mathrm{~m}$. 
orientación de $\mathrm{N} 023^{\circ}$ y una inclinación de $72^{\circ} \mathrm{SE}$. Corta toda la secuencia hasta la unidad 4 pero sin alcanzar el suelo reciente. La falla presenta movimiento normal con el bloque de techo cayendo al sureste. En sentido vertical, esta falla desplaza la unidad 3 un total de $8 \pm 3 \mathrm{~cm}$, mientras que en sentido horizontal se abre hasta $3 \mathrm{~cm}$ y se rellena de pómez fibrosa.

Asociadas a F2 y f2a se observan abundantes fracturas conjugadas que aparentemente no desplazan las capas de la secuencia estratigráfica en sentido vertical. Sin embargo, algunas de estas presentan apertura horizontal menor a $3 \mathrm{~cm}$.

La tercera falla principal (F3, en la Figura 9) es una estructura antitética a F2 pero sintética a $\mathrm{F} 1$, que presenta una orientación de $\mathrm{N} 245^{\circ}$ y una inclinación de $83^{\circ} \mathrm{NO}$. Esta falla presenta movimiento normal con el bloque de techo cayendo hacia el noroeste. El desplazamiento vertical observable en la unidad 2 es de $11 \pm 4 \mathrm{~cm}$ en total.
Su apertura horizontal máxima es de $5 \mathrm{~cm}$ y se rellena por pómez perteneciente a la unidad 4 (Figura 9). Asociadas a F3, se observan al menos 7 fracturas adicionales, prácticamente con la misma orientación e inclinación. La mayoría de estas estructuras cortan toda la secuencia estratigráfica sin generar desplazamientos verticales pero sí aperturas horizontales de hasta $4 \mathrm{~cm}$ rellenas de pómez. La excepción es la falla f3a que desplaza verticalmente a la unidad 3 , aproximadamente 3 $\pm 1 \mathrm{~cm}$.

En la pared E-O se observa también la formación de un pequeño graben delimitado por las fallas identificadas como F7, F8 y F9, con orientaciones e inclinaciones de $\mathrm{N} 188^{\circ} / 78^{\circ} \mathrm{O}, \mathrm{N} 221^{\circ} / 82 \mathrm{SE}$ y $\mathrm{N} 015^{\circ} / 80^{\circ} \mathrm{E}$, respectivamente. La falla $\mathrm{F} 7$ corta la secuencia estratigráfica hasta la superficie, pero no genera un escarpe, el plano de la falla ocasiona un desplazamiento vertical de $18 \pm 6 \mathrm{~cm}$ en la unidad 3, en cambio las fallas F8 y F9 afectan únicamente
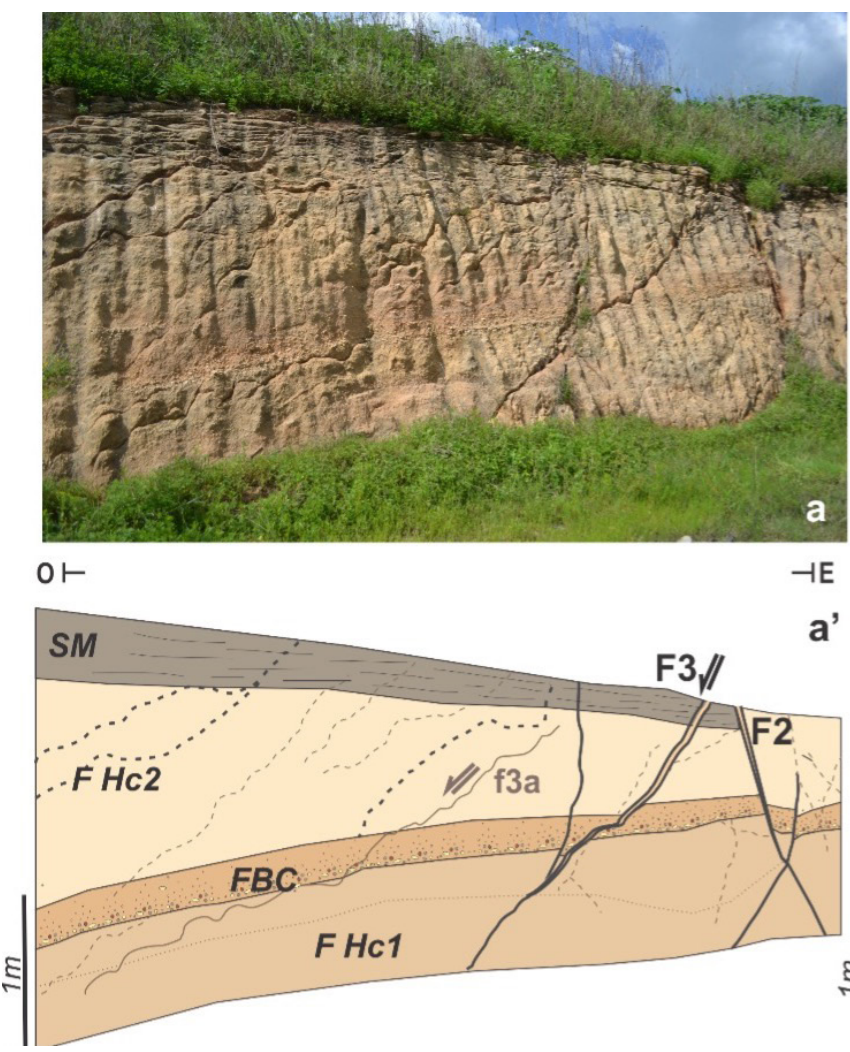

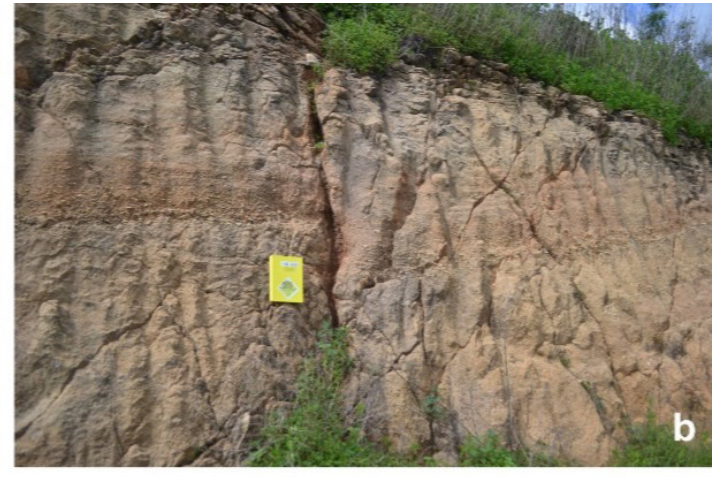

oト

F2

-IE

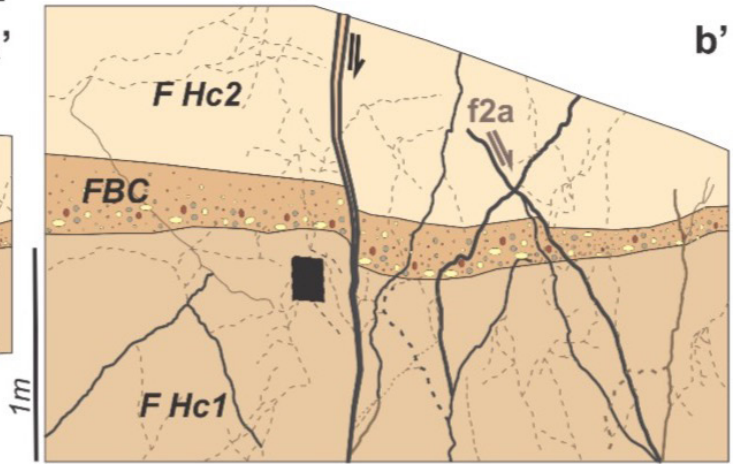

Figura 9 (a, b) Fotografías e (a', b') interpretación de las estructuras F2 y F3 en el afloramiento A1, así como las estructuras secundarias asociadas. 
a las unidades 2 y 3 (DCG y FBC), ocasionando un desplazamiento vertical de la unidad FBC de $3 \pm 1$ y $22 \pm 8 \mathrm{~cm}$, respectivamente (Figura 10).

\subsubsection{DEFORMACIÓN EN LA SECCIÓN A2}

En esta pared se observan dos fallas principales que afectan toda la secuencia (F4 y F5), pero al igual que las anteriores no generan escarpes superficiales visibles y se comportan como fracturas rellenándose de materiales de las unidades litológicas superiores, por lo que se clasifican también como fallas mixtas. Asimismo, se observan dos fallas secundarias que solo son distinguibles en las unidades superiores de la secuencia, pues no es apreciable su propagación en profundidad (Figura 10). La falla principal (F4, en la Figura 10) presenta desplazamiento de tipo normal con el bloque de techo cayendo hacia el noroeste. Posee una orientación $\mathrm{N} 240^{\circ}$ e inclinación de $56^{\circ} \mathrm{NO}$. Esta falla desplaza verticalmente a la unidad estratigráfica 3 (DCG) un total de $84 \pm 14 \mathrm{~cm}$; presenta una apertura en sentido horizontal que va de $4 \mathrm{~cm}$
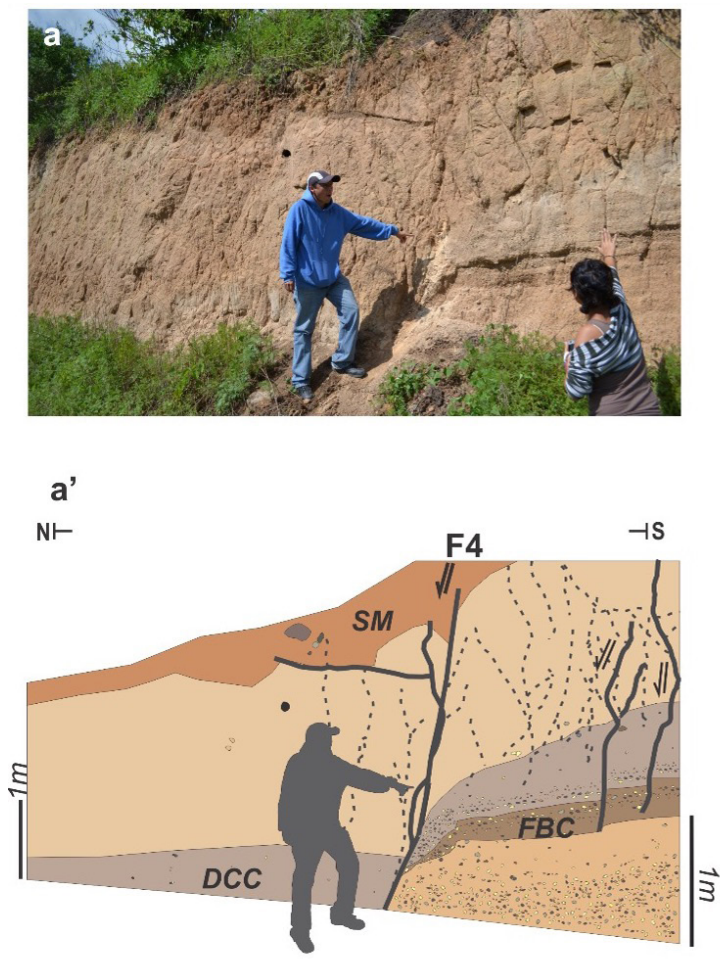

en la base, ensanchándose hacia la cima donde alcanza $30 \mathrm{~cm}$ (Figura 10). Esta falla se rellena de material procedente de la unidad 4.

La segunda falla en importancia (F5, en la Figura 10) es normal con el bloque de techo cayendo hacia el noroeste, posee una orientación de $\mathrm{N} 252^{\circ}$ e inclinación de $89^{\circ} \mathrm{NO}$. Esta falla se ubica muy cerca de la esquina del afloramiento, donde se unen las paredes norte-sur y este-oeste. El plano de falla desplaza a las unidades DCG y FBC un total de $10 \pm 3 \mathrm{~cm}$ en la vertical, pero el desplazamiento máximo total de esta unidad se obtuvo al combinar el desplazamiento que generan las fallas secundarias asociadas f5a y f5b $\left(\mathrm{N} 250^{\circ} / 75^{\circ} \mathrm{NO}\right.$ y $\mathrm{N} 245^{\circ} / 71^{\circ} \mathrm{NO}$ ), sumando un total de $24 \pm 8 \mathrm{~cm}$. En la horizontal presentan aperturas de $1 \mathrm{a} 4 \mathrm{~cm}$ y se rellenan de material perteneciente a la unidad 4 (Figura 10).

Se considera que la falla El Malpaso - El Salto está representada por los planos de falla F4 y F5 y que su desplazamiento vertical total puede ser obtenido mediante la suma de los desplazamientos parciales de las mismas $(84 \pm 14+24 \pm 8=108 \pm 22$
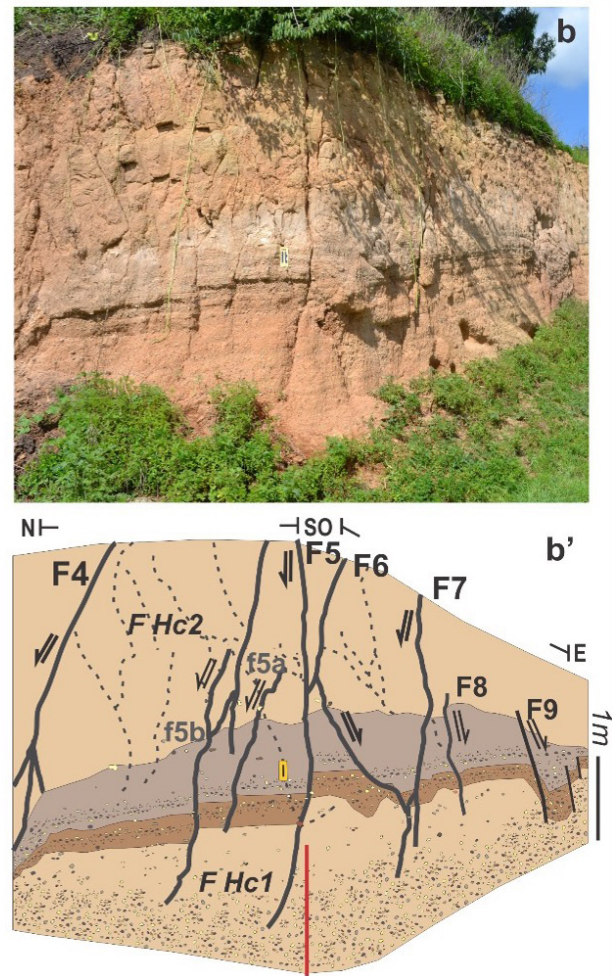
cm). Mientras que las fallas F1 y F3 son las estructuras de relevo orientadas al NE-SO. Asociadas a F4 y F5 se observa una serie de pequeñas fracturas que no parecen afectar de manera vertical las capas de la secuencia, pero sí presentan aperturas horizontales menores a $2 \mathrm{~cm}$.

Una falla relacionada a F5 y que aparece en una geometría antitética a ésta, fue identificada como F6 y posee una orientación de $\mathrm{N} 046^{\circ}$ e inclinación de $88^{\circ}$ al SE (Figura 10). Igual que F5 esta falla se ubica sobre la esquina del afloramiento, un poco más desplazada hacia el borde oeste de la pared este-oeste. Esta falla desplaza la unidad FBC un total de $22 \pm 7 \mathrm{~cm}$ en la vertical, distribuido en dos escalones de $13 \pm 4$ y $9 \pm 3 \mathrm{~cm}$, respectivamente.

\section{Estimaciones de la actividad de la falla El Malpaso - El Salto}

\subsection{TASAS DE DESPLAZAMIENTO (SR)}

Debido a la escasez de material fechable en el afloramiento, únicamente se calculó la tasa de desplazamiento vertical a largo plazo para la FMAS considerando el escarpe acumulado máximo (120 $\mathrm{m}$ con un error de $\pm 15 \mathrm{~m}$ ) y la edad publicada para la secuencia estratigráfica expuesta en el afloramiento $(1.4 \pm 0.07 \mathrm{Ma}$, de acuerdo con Kshirsagar et al., 2015). El SR obtenido fue de $0.086 \pm$ $0.015 \mathrm{~mm} /$ año, lo cual representa un poco más de la mitad del SR estimado por Suter et al., (2001) de $0.16 \mathrm{~mm} / \mathrm{año}$, mediante las unidades litológicas (ignimbritas pliocénicas y basaltos de 0.87-0.75 Ma del Lago de Cuitzeo y andesitas/basaltos del Mioceno Tardío de Tarímbaro) afectadas por los escarpes con saltos de $\sim 100 \mathrm{~m}$.

En el caso de la componente de desplazamiento lateral izquierdo observable en la morfología, no se encontraron marcadores que nos permitieran realizar una estimación de la cantidad de movimiento horizontal asociada a la falla, sin embargo, para esta zona Pérez-Orozco et al., (2018) reporta un sitio donde se observa movimiento horizontal en el rango de 10 a $40 \mathrm{~cm}$ afectando las unidades de edades de $1.4 \pm 0.07 \mathrm{Ma}$ (Kshirsagar et al., 2015).
Con estos datos se puede hacer el ejercicio de estimar burdamente una tasa de desplazamiento horizontal preliminar ( $\leq 0.0002 \mathrm{~mm} /$ año), sin embargo, la incertidumbre de esta sería muy elevada.

Los valores estimados de SR para la FMAS aunque preliminares, son similares a las tasas de desplazamiento obtenidas para otras fallas que conforman el SFMA, especialmente dentro de la región de Morelia - Cuitzeo (entre 0.05 y 0.18 mm/ año; Suter et al., 2001). Para las fallas en los extremos del SFMA, se han estimado SR más variables, reportándose rangos entre 0.03 y $2 \mathrm{~mm} /$ año para la zona de Acambay y entre 0.009 y $2.78 \mathrm{~mm} /$ año para la región de Pátzcuaro (donde se incluyen estimaciones de largo y corto plazo) (Suter et al., 1992; Langridge et al., 2000 y 2013; Garduño-Monroy et al., 2009; Sunye-Puchol et al., 2015; Ortuño et al., 2015 y 2019; Suter, 2016; Lacan et al., 2018).

\subsection{PERÍODOS O INTERVALOS DE REGURRENGIA (RI)}

Para estimar el período de recurrencia de la actividad de la FMAS, se consideró el desplazamiento vertical sintético $(108 \mathrm{~cm})$ menos el desplazamiento antitético $(22 \mathrm{~cm})$ obtenidos de las mediciones en el afloramiento y el error promedio $( \pm 9 \mathrm{~cm}) . \mathrm{El}$ resultado obtenido fue de $86 \pm 9 \mathrm{~cm}$ para el desplazamiento vertical de la falla. Para todos los cálculos se asume que el desplazamiento por creep es nulo $(\mathrm{C}=0)$.

Se estimaron períodos de recurrencia de 10021 \pm 1221 años y $12580 \pm 1266$ años, considerando el desplazamiento sintético menos el valor antitético y solo el valor sintético, respectivamente. Estos valores son similares a los intervalos de recurrencia calculados para otros segmentos del SFMA como son la falla Pastores $(10$ - 15 mil años de acuerdo con Langridge et al., 2013; Ortuño et al., 2015) y la falla San Mateo (1 $1570 \pm 5320$ años de acuerdo con Sunye-Puchol et al., 2015). Las fallas de la región de Pátzcuaro - Morelia, el valor es cercano al de la falla La Alberca-Teremendo (7726 años), pero otras fallas (p.e. falla La Paloma, Huiramba, Charo y Queréndaro-Indaparapeo) presentan períodos más variables (4.5 a 20 mil años; Garduño-Monroy et al., 2009). 
Tabla 3. Relaciones empíricas resueltas para la evaluación de la magnitud MW potencial para un evento máximo de ruptura en la falla Malpaso - El Salto (FMAS).

\begin{tabular}{|l|l|l|}
\hline EVENTO & RELACIONES USADAS & MAGNITUDES CALCULADAS $\left(\boldsymbol{M}_{\mathbf{w}}\right)$ \\
\hline $\begin{array}{l}\text { Ruptura máxima para } \\
\text { la longitud total } \\
\text { (LRS) de la FMAS }\end{array}$ & Wells y Coppersmith (1994) & 6.77 \\
& Anderson et al., (1996) & $6.66-7.18$ \\
\hline & Stirling et al., (2002) & $6.73-7.36$ \\
\hline & Wesnousky (2008) & 6.80
\end{tabular}

Los períodos de recurrencia aquí reportados para evaluar la actividad de la falla El Malpaso - El Salto son preliminares y deben ser tomados con precaución. Es posible apreciar que la ocurrencia de sismos con magnitudes altas puede estar agrupada en el tiempo, con pocos años o inclusive días de separación, como se ha observado en varias partes del mundo (Iezzi et al., 2018). Para la frecuencia de eventos que pueden ocurrir en la FMAS, también se debe considerar la influencia de la actividad magmática de la región, lo cual puede cambiar el estado del campo de esfuerzos en torno a la falla.

\subsection{MAGNITUD POTENGIAL MÁXIMA ESPERADA PARA UN EVENTO DE RUPTURA DE LA FMAS}

Si se asume que la FMAS se mueve de manera sísmica y los segmentos que la conforman están conectados en profundidad, produciendo una longitud total de la falla de $28.874 \mathrm{~km}$ (LRS, con un error asociado de $\pm 15 \mathrm{~m}$ ), entonces los valores de magnitud de momento $M_{\mathrm{w}}$ varían entre 6.66 y 7.36. Estos valores se encuentran dentro de los rangos máximos reportados para otras fallas del SFMA, por ejemplo: los valores de 6.4 a 7 para la región del graben de Acambay (fallas Pastores, Acambay-Tixmadejé, Maravatío, Venta de Bravo y San Mateo), y los valores de 5.8 a 7.1 obtenidos para las fallas de la región Pátzcuaro-Morelia (Langridge et al., 2000 y 2013; Garduño-Monroy et al., 2009; Ortuño et al., 2015; Suter, 2016; Lacan et al., 2018, estos trabajos usan las relaciones de Wells y Coppersmith, 1994; Wesnousky, 2008).

Las magnitudes máximas $M_{\mathrm{w}}$ obtenidas para esta falla usando las relaciones de Anderson et al. (1996) y Stirling et al. (2002), son mayores que las que se obtuvieron usando las relaciones de Wells y Coppersmith (1994) y Wesnousky (2008); sin embargo, la magnitud mínima estimada para una ruptura de $28 \mathrm{~km}$ de la FMAS se mantiene alrededor 6.7 (Tabla 3).

Las magnitudes máximas potenciales estimadas para la FMAS son relativamente altas considerando que se trata de una falla con longitud de 28 $\mathrm{km}$, y que se han obtenido magnitudes semejantes para otras fallas del SFMA con longitudes cercanas a los $50 \mathrm{~km}$ (p.e. las fallas de Acambay-Tixmadejé, Venta de Bravo o Pastores).

En términos generales las fallas del SFMA responden al campo de esfuerzos transtensivo actual, sin embargo, las estructuras del sector este (Acambay) reflejan condiciones locales distintas a las del sector oeste (Morelia-Cuitzeo), ambos sectores son separados además por una frontera regional representada por el anticlinal de Tzitzio (Figura 1; Mennella et al., 2000).

Las fallas del sector oeste coexisten con escenarios de volcanismo monogenético muy abundante del Pleistoceno - Holoceno, así como con el límite suroeste del SFZQ o la zona de fallas Zacapu-Salvatierra (Suter et al., 2001; Suter, 2002; Quintero-Legorreta, 2002). En esta zona la extensión parece ser acomodada tanto por el alineamiento de los edificios y depósitos volcánicos como por la actividad de ruptura de las fallas ENE. Esto puede afectar las estimaciones de desplazamientos verticales y magnitudes máximas potenciales para el sector oeste.

En este contexto, la FMAS podría moverse como respuesta a un evento volcano-tectónico o puramente tectónico, ya sea como una ruptura 
primaria o bien como una ruptura secundaria detonada por cambios en el campo de esfuerzos locales producto de una ruptura en fallas vecinas a ella y pertenecientes al mismo sistema. Particularmente, la falla La Alberca-Teremendo (FLAT, perteneciente al SFMA; Figuras 4 y 5), localizada a escasos 2.5-4 km al sureste de la FMAS, posee una geometría y cinemática muy parecida (falla normal con una ligera componente de desplazamiento lateral).

La FLAT es una falla con orientación $\mathrm{N} 260^{\circ} / 76^{\circ} \mathrm{NO}, 60^{\circ}$ pitch y geometría compleja, se compone por 11 segmentos acomodados en échelon que sumados alcanzan una longitud de $\sim 26 \mathrm{~km}$, las estimaciones de potencialidad indican que tiene la capacidad de generar rupturas asociadas a magnitudes $M_{\mathrm{w}}$ entre 6.7 y 7.3 , mientras que el estudio de trincheras muestra que esta falla ha generado al menos 3 rupturas en los últimos 23000 años, con el evento más reciente afectando los suelos modernos. Para esta falla se estiman valores SR de $0.11 \mathrm{~mm} /$ año y períodos de recurrencia de $7726 \pm 68$ años (Soria-Caballero et al., 2019).

\section{Evaluación de la deformación observada en afloramiento}

Del análisis de las secciones del afloramiento se obtuvo información acerca de las fases de deformación que pudieron haber afectado a las secuencias piroclásticas septentrionales del Complejo Volcánico El Tzirate. En la sección Al se observa que las fallas principales corresponden a F1, F2 y F3, las cuales pertenecen a la familia de fallas principal con dirección NE y son consideradas estructuras de relevo, siendo F1 y F3 sintéticas y F2 antitética a ellas (Figuras 8 y 9). El acomodo de estas fallas muestra geometrías de horst-graben, muy marcadas. En las unidades estratigráficas afectadas por estas fallas se puede apreciar que la unidad 3 (depósito de caída de cenizas) no aparece claramente en la secuencia, en su lugar se observa que la unidad 2 (el flujo de bloques y cenizas) se encuentra en contacto directo con el flujo hiperconcentrado más reciente (unidad 4), el cual es cubierto por el suelo actual. En consecuencia el desplazamiento de las unidades estratigráficas solo pudo medirse sobre la unidad 2 (FBC).

El desplazamiento sintético para la unidad 2 (FBC) se obtuvo sumando el desplazamiento sobre F1 $(32 \pm 9 \mathrm{~cm})$ y F3 $(14 \pm 9 \mathrm{~cm})$, lo cual resulta en $46 \pm 9 \mathrm{~cm}$. Si a este valor se le resta el desplazamiento antitético para la misma unidad registrado en F2 (45 $\pm 9 \mathrm{~cm})$ se obtiene una diferencia de 1 $\mathrm{cm}$. Este resultado sugiere un balance en los movimientos de las fallas normales, donde, después de la deposición de la secuencia piroclástica del CVT, ocurrió un evento de extensión que generó las geometrías de graben-horst-graben que pueden observarse en esta sección. Respecto a las fallas secundarias F7, F8 y F9, aunque afectan visiblemente solo la unidad FBC y sus terminaciones no son claramente visibles en las unidades de la base y la cima de la secuencia, se comportan de manera similar a las tres anteriores (Figura 10), con F7 como estructura sintética, F8 como antitética y F9 como estructura de bajo ángulo con respecto a la familia de fallas principales (NE - SO). Estas fallas forman geometrías de horst-semigraben-graben, de menores dimensiones que las formadas por las fallas F1 a F3. Igualmente, la magnitud de sus desplazamientos está compensada sugiriendo el evento de extensión, pero también la ocurrencia de transtensión sugerida por la dirección de F9. Este evento de extensión podría corresponder a la fase extensiva que reporta Mennella et al. (2000) para el Pleistoceno Superior - Holoceno.

En la sección A2 se observa que la deformación más importante está representada por los desplazamientos verticales más grandes medidos para una unidad estratigráfica (en este caso la unidad 3: el depósito de caída de ceniza). Aquí, el desplazamiento se acomoda a través de los planos de F4 (84 $\pm 9 \mathrm{~cm})$, F5 $(24 \pm 9 \mathrm{~cm})$ y pequeñas fallas secundarias paralelas a estas, las cuales comparten dirección e inclinación. Hacia el extremo sur de A2 aparece la falla F6 $(22 \pm 9 \mathrm{~cm})$ que puede considerarse antitética a F5.

La zona de fallamiento generada por estas estructuras se considera correspondiente al área 
de cizalla principal ocasionada por la FMAS, reflejando un desplazamiento vertical sintético de $108 \pm 9 \mathrm{~cm}$ y un desplazamiento vertical sintético menos antitético de $86 \pm 9 \mathrm{~cm}$. Las dimensiones de este desplazamiento sugieren un movimiento tectónico de la FMAS ocurrido posteriormente al evento de extensión que produjo las morfologías de horst-graben, aunque se requiere más trabajo para determinar si este desplazamiento fue producido por uno o más eventos sísmicos.

Si bien las relaciones empíricas sugieren que la FMAS tiene la capacidad para producir sismos de magnitudes altas, la escasez de edades para las unidades del afloramiento y la falta de otros marcadores tales como suelos fallados limitan las estimaciones aquí presentadas.

\section{La interacción de la falla El Malpaso-El Salto y otras estructuras del SFMA}

La falla El Malpaso-El Salto es una de las estructuras más sobresalientes del norte de Michoacán debido a sus dimensiones (longitud de $28 \mathrm{~km}$ y escarpe acumulado de hasta $120 \mathrm{~m}$ ). Posee características geométricas (orientación e inclinación de $\mathrm{N} 250^{\circ} / 56^{\circ}-75^{\circ} \mathrm{NW}$, rotación del bloque caído de hasta $12^{\circ}$, evidencias morfológicas que indican la presencia de una ligera componente de desplazamiento lateral izquierdo) que la hacen muy similar a su vecina ubicada al sur, la falla La Alberca-Teremendo (FLAT, Figuras 4 y 5), cuya actividad paleosísmica fue estudiada recientemente (Soria-Caballero et al., 2019). Estas fallas se consideran geométrica y cinemáticamente similares de acuerdo con los criterios propuestos en Quigley et al., (2017) (cambios en dirección e inclinación del plano de falla menores a $20^{\circ}$ y en el vector de deslizamiento menor a $30^{\circ}$ ). Ambas fallas poseen magnitudes potenciales $M_{\text {w }}$ de hasta 7 y desplazamientos verticales por evento cercanos al metro. Aunque existen diferencias en sus períodos de recurrencia $(10021 \pm 1221$ años y $7726 \pm 68$ años, respectivamente) y tasas de deslizamiento $(0.086 \pm 0.015 \mathrm{~mm} /$ año y $0.11 \mathrm{~mm} /$ año, respectivamente), los valores no están muy alejados entre sí. Un resumen de los parámetros importantes para la FMAS se encuentra en la tabla complementaria 2.

$\mathrm{Al}$ igual que la FLAT, la FMAS se encuentra ubicada en la porción oeste del SFMA, cerca de la frontera con el sistema de fallas Zacapu-Querétaro (zona de fallas Zacapu-Salvatierra, Suter et al., 2001) y dentro del área de evolución del campo volcánico Michoacán-Guanajuato; la FLAT posee una orientación $\left(\mathrm{N} 250^{\circ}\right)$ que es casi perpendicular a la dirección de la extensión del campo de esfuerzos actual (NO), lo cual genera escenarios propicios para que la falla genere rupturas derivadas tanto de la influencia del ambiente volcánico activo, como del régimen tectónico actual.

Ambas fallas son paralelas entre sí, la FMAS se encuentra a menos de $3 \mathrm{~km}$ de distancia de la FLAT dentro del área de influencia del bloque caído de esta última, y si se considera la geometría lístrica que se ha reportado para las estructuras de esta zona, es posible sugerir una posible conexión en profundidad de ambas fallas.

De acuerdo con Wesnousky (2008), las rupturas asociadas a sismos con magnitudes altas pueden propagarse desde la falla de origen hasta otras trazas adyacentes, debido a que se producen cambios en los esfuerzos locales que pueden detonar desplazamientos en fallas que se encuentren a distancias entre 3-4 km (escenarios de fallamiento lateral) hasta 5-7 km (escenarios de fallamiento normal).

Esto implica que en áreas tectónicamente activas que incluyan fallas con geometrías complejas, ya sea en échelon o con trazas paralelas y subparalelas, es necesario considerar la distribución de esos segmentos para lograr una estimación confiable del peligro sísmico.

En el contexto tectónico del norte de Michoacan, la FMAS y la FLAT podrían interaccionar debido a cambios en el estado de esfuerzos local, dichos cambios podrían ser detonados por alguno de los segmentos. Sin embargo, más estudios son necesarios para clarificar la dinámica entre estas fallas y refinar las estimaciones de las tasas de desplazamiento y períodos de recurrencia asociadas a dicha interacción. 
Es probable que las magnitudes preliminares estimadas en este trabajo cobren más sentido con las nuevas definiciones de subsistemas de fallamiento de la cuenca del Lago de Cuitzeo propuestos en el trabajo de Gómez-Vasconcelos et al., (2021), en donde la FMAS y la FLAT son las trazas maestras de una zona de cizalla de $\sim 30 \mathrm{~km}$ de largo y $\sim 10 \mathrm{~km}$ de ancho, que incluye más de 200 trazas secundarias asociadas a ellas.

Las interacciones entre fallas cercanas, produciendo incluso rupturas multi-falla han sido reportadas para las fallas de la porción oriental del SFMA, donde han jugado un papel muy importante en la reactivación de trazas (Arzate et al., 2018; Ortuño et al., 2019), incrementando las magnitudes y los desplazamientos verticales ocasionados por eventos individuales; sin embargo, en la porción occidental del sistema este tema no ha sido abordado, por lo cual las estimaciones de actividad tectónica en la zona podrían ser menores a las reales.

Es necesaria la realización de estudios detallados en esta región del SFMA a fin de puntualizar las similitudes y diferencias entre los sectores que lo conforman y proponer relaciones empíricas que consideren las particularidades de dichas zonas.

\section{Consideraciones finales}

La falla El Malpaso-El Salto es una de las más largas del norte de Michoacán y del Sistema Morelia-Acambay. Su morfología y geometría sugiere una actividad continua al menos desde el Mioceno Tardío, con evidencias de desplazamientos de $\sim 80$ $\mathrm{cm}$ afectando unidades litológicas con edades de $\sim 1$ Ma.

Para esta falla se estimó una tasa de deslizamiento preliminar de $0.086 \pm 0.015 \mathrm{~mm} /$ año y un período de recurrencia preliminar entre $10021 \pm 1221$ y 12606 \pm 2278 años. Esta falla se asocia estrechamente a la falla La Alberca-Teremendo, la cual presenta evidencias de movimiento durante los últimos 23000 años, siendo el evento más reciente hace menos de mil años. Las dimensiones y potencialidad calculadas para ambas fallas es similar, su orientación paralela y su geometría lístrica sugieren la posibilidad de una conexión a profundidad de los planos de falla, lo que podría derivar en interacciones entre ellas. Bajo estas condiciones, la zona de cizallamiento delimitada por la FMAS y la FLAT podría generar sismos con magnitudes $M_{w}$ de 6.7 hasta 7.3 , lo que la convierte en un foco de atención para las evaluaciones de riesgo sísmico en el centro de México. Los resultados aquí presentados son una primera aproximación a la evaluación de la actividad de la falla El Malpaso-El Salto de la cual se tenían muy pocos datos; sin embargo, están restringidos por la naturaleza del afloramiento analizado, la falta de fechamientos de los materiales expuestos y la problemática social local que dificultan la obtención de los permisos necesarios para realizar un trabajo en campo más detallado. Para refinar las estimaciones aquí presentadas, se requiere más trabajo paleosismológico, geofísico y geológico-estructural en el área.

\section{Agradecimientos}

Los resultados de este trabajo forman parte del estudio doctoral de la autora, el cual fue realizado con el apoyo de la beca No. 59131 otorgada por el Consejo Nacional de Ciencia y Tecnología (CONACyT), así como el apoyo del proyecto SEP-CONACyT-GB-2009-01-134151 titulado "Estudio tectónico, paleosismológico y arqueosismológico en lagos del Holoceno al Reciente del Cinturón Volcánico Trans-Mexicano y el Bloque Jalisco", y el proyecto 17 del Cemie-GEO, ambos a cargo del Dr. Víctor Hugo Garduño-Monroy. Los autores agradecen las observaciones y sugerencias de los revisores Dra. María Ortuño Candela y Dr. Luis Martin Rothis, así como de Dra. Laura Perucca, los cuales mejoraron significativamente la calidad del presente manuscrito. Los autores agradecen también el apoyo de la Escuela Nacional de Estudios Superiores campus Morelia, particularmente al grupo de la licenciatura en Geociencias. Este trabajo se dedica al muy extrañado Dr. Víctor Hugo Garduño, quien supo infundir la pasión por la Geología en las jóvenes mentes que se cruzaron por su camino. 


\section{Referencias}

Anderson, J.G., Wesnousky, S.G., Stirling, M. 1996, Earthquake size as a function of fault slip rate: Bulletin of the Seismological Society of America, 86 (3), 683-690.

Arzate, J., Lacan, P., Corbo-Camargo, F., ArangoGalván, C., Felix-Maldonado, R., Pacheco, J., León-Loya, R. 2018, Crustal structure of the eastern Acambay Graben, Central Mexico, from integrated geophysical data: Revista Mexicana de Ciencias Geológicas, 35(3),228-239. https://doi.org/10.22201/ cgeo.20072902e.2018.3.864

Avellán, D.R., Cisneros-Máximo, G., Macías, J.L., Gómez-Vasconcelos, M.G., Layer, P.W., Sosa-Ceballos, G., Robles-Camacho, J. 2020, Eruptive chronology of monogenetic volcanoes northwestern of Morelia-Insights into volcano-tectonic interactions in the centraleastern Michoacán-Guanajuato Volcanic Field, México: Journal of South American Earth Sciences, 100, 102554. https://doi. org/10.1016/j.jsames.2020.102554

Ego, F., Ansan, V., 2002, Why is the Central TransMexican Volcanic Belt $\left(102^{\circ}-99^{\circ} \mathrm{W}\right)$ in transtensive deformation?: Tectonophysics, 359, 189-208. https://doi.org/10.1016/ s0040-1951(02)00511-5

García-Palomo, A., Macías, J.L., Garduño, V.H., 2000, Miocene to Recent structural evolution of the Nevado de Toluca volcano region, central Mexico: Tectonophysics, 318 (1), 281-302. https://doi.org/10.1016/ s0040-1951(99)00316-9

Garduño-Monroy, V. H., Pérez-López, R., IsradeAlcántara, I., Rodríguez-Pascua,M.A., Szynkaruk, E., Hernández-Madrigal,V.M., García-Zepeda,M.L.,Corona-Ghávez, P., Ostroumov,M.Medina-Vega,V.H., García-Estrada, G., Carranza, O., LópezGranados, E., Mora Chaparro, J. C., 2009, Paleoseismology of the southwestern MoreliaAcambay fault system, central Mexico: Geofisica Internacional, 48(3), 319-335.
Gómez-Vasconcelos, M.G., Macías, J., Avellán, D.R., Sosa-Geballos, G., Garduño-Monroy, V.H., Cisneros-Máximo, G., Layer, P.W., Benowitz, J., López-Loera, H., López, F.M., Perton, M., 2020, The control of preexisting faults on the distribution, morphology, and volume of monogenetic volcanism in the Michoacán-Guanajuato Volcanic Field: Geological Society of America Bulletin, 132(11-12), 2455-2474. https://doi. org/10.1130/b35397.1

Gómez-Vasconcelos, M. G., Avellán, D. R., SoriaCaballero, D., Macías, J. L., Velázquez-Bucio, M. M., Jiménez-Haro, A., Israde-Alcántara, I., Garduño-Monroy, V.H., Ávila-Olivera, J.A., Figueroa-Soto, A.G., Cisneros-Máximo, G., Cardona-Melchor, S., 2021, Geomorphic characterization of faults as earthquake sources in the Cuitzeo Lake basin, central México: Journal of South American Earth Sciences, 109, 103196. https://doi. org/10.1016/j.jsames.2021.103196

Iezzi, F., Mildon, Z., Walker, J. F., Roberts, G., Goodall, H., Wilkinson, M., Robertson, J., 2018, Coseismic throw variation across along-strike bends on active normal faults: Implications for displacement versus length scaling of earthquake ruptures: Journal of Geophysical Research: Solid Earth, 123(11), 9817-9841. https://doi. org/10.1029/2018jb016732

Instituto Nacional de Estadística y Geografía (INEGI), 2010, Cartas topográficas 1:50000, Coeneo de la Libertad, Morelia y Patzcuaro. Disponible en: http://www.inegi.org.mx

Instituto Nacional de Estadística y Geografía (INEGI), 2010, Fotografías aéreas 1:20000, Coeneo de la Libertad. Disponible en: http://www.inegi.org.mx

Instituto Nacional de Estadística y Geografía (INEGI), 2010, Modelo LIDAR $15 \mathrm{~m}$ de resolución, Morelia. Disponible en: http:// www.inegi.org.mx

Israde-Alcántara,I., Garduño-Monroy, V.H., 1999, Lacustrine record in a volcanic intra-arc 
setting. The evolution of the Late Neogene Cuitzeo basin system (Central western Michoacan, Mexico): Palaeogeography, Palaeoclimatology, Palaeoecology, 151(1), 209-227.https://doi.org/10.1016/ s0031-0182(99)00024-3

Israde Alcántara, I., Velázquez-Durán, R., Lozano García, M., Bischoff, J., Domínguez Vázquez,G., Garduño Monroy, V.H., 2010, Evolución paleolimnológica del Lago Cuitzeo, Michoacán durante el PleistocenoHoloceno: Boletín de la Sociedad Geológica Mexicana, 62 (3), 345-357. https://doi. org/10.18268/bsgm2010v62n3a3

Kshirsagar, P., Siebe, G., Guilbaud, M. N., Salinas, S., Layer, P. W., 2015, Late Pleistocene Alberca de Guadalupe maar volcano (Zacapu basin, Michoacán): Stratigraphy, tectonic setting, and paleo-hydrogeological environment: Journal of Volcanology and Geothermal Research, 304, 214-236. https://doi. org/10.1016/j.jvolgeores.2015.09.003

Lacan, P., Ortuño, M., Audin, L., Perea, H., Baize,

S., Aguirre-Díaz, G., Zúñiga, F. R., 2018, Sedimentary evidence of historical and prehistorical earthquakes along the Venta de Bravo Fault System, Acambay Graben (Central Mexico): Sedimentary Geology, 365, 62-77. https://doi.org/10.1016/j. sedgeo.2017.12.008

Lafuente Tomás, P., 2011, Tectónica activa y paleosismicidad de la falla de Concud (Cordillera Ibérica central). Universidad de Zaragoza, Tesis doctoral, $274 \mathrm{p}$.

Langridge, R.M., Weldon II, R.J., Moya, J.G., Suárez, G., 2000, Paleoseismology of the 1912 Acambay earthquake and the AcambayTixmadejé Fault, Trans-Mexican Volcanic Belt: Journal of Geophysical Research, Solid Earth (1978-2012), 105 (B2), 3019-3037. https://doi.org/10.1029/1999jb900239

Langridge, R.M., Persaud, M., Zúñiga, F.R., Aguirre-Díaz, G.J., Villamor, P., Lacan, P., 2013, Preliminary paleoseismic results from the Pastores fault and its role in the seismic hazard of the Acambay graben,
Trans-Mexican Volcanic Belt, Mexico: Revista Mexicana de Ciencias Geológicas. 30 (3), $463-481$.

Martínez-Reyes, J., Nieto-Samaniego, A.F., 1990, Efectos Geológicos de la Tectónica Reciente en la Parte Central de México: Revista Mexicana de Ciencias Geológicas, 9 (1), 33-50.

McCalpin, J.P., 1996, Paleoseismology. Academic Press, San Diego, California, 588 p.

Mendoza-Ponce, A., Figueroa-Soto, A., SoriaCaballero,D., Garduño-Monroy, V. H., 2018, Active faults sources for the PátzcuaroAcambay fault system (Mexico): fractal analysis of slip rates and magnitudes Mw estimated from fault length: Natural Hazards \& Earth System Sciences, 18(11), 3121-3135. https://doi.org/10.5194/ nhess-18-3121-2018

Mennella, L., Garduño, V.H., Bonassi, O., 2000, Fault-slip analysis in the basal units of the Mexican Volcanic Belt on the eastern flank of the Tzitzio anticline, Michoacán, México. In Delgado-Granados, H., Aguirre-Díaz, G., and Stock, J.M., eds., Cenozoic Tectonics and Volcanism of Mexico: Boulder, Colorado: Geological Society of America Special Paper 334, 237-246. https://doi. org/10.1130/0-8137-2334-5.237

Mennella, L., 2011, Sismotectónica del sector occidental del Sistema Morelia-Acambay, México, a partir del análisis de poblaciones de fallas. Universidad Michoacana de San Nicolás de Hidalgo Tesis de Maestría,151 p.

Ortuño, M., Zúñiga, F. R., Aguirre-Díaz, G. J., Carreón-Freyre, D., Cerca, M., Roverato, M., 2015, Holocene paleo-earthquakes recorded at the transfer zone of two major faults: The Pastores and Venta de Bravo faults (Trans-Mexican Volcanic Belt): Geosphere, 11(1), 160-184. https://doi.org/10.1130/ GES01071.1

Ortuño, M., Corominas, O., Villamor, P., Zúñiga, R. F., Lacan, P., Aguirre-Díaz, G., Perea, H., Štěpančíková, P., Ramírez-Herrera, M. T., 2019, Evidence of recent ruptures 
in the central faults of the Acambay Graben (central Mexico): Geomorphology, 326, 17-37. https://doi.org/10.1016/j. geomorph.2018.07.010

Pérez-Orozco, J.D., Sosa-Ceballos, G., GarduñoMonroy,V.H., Avellán,D.R., 2018, Felsicintermediate magmatism and brittle deformation in Sierra del Tzirate (MichoacánGuanajuato Volcanic Field): Journal of South American Earth Sciences, 85, 81-96. https:// doi.org/10.1016/j.jsames.2018.04.021

Quigley, M., Mohammadi, H., Jiménez, A. y Duffy, B., 2017, Multi-fault earthquakes with kinematic and geometric rupture complexity: how common? In: 8th International INQUA Meeting on Paleoseismology, Active Tectonics and Archeoseismology (PATA), 13 - 16 November, 2017, New Zealand, INQUA Focus Group Earthquake Geology and Seismic Harzards, 316 - 318.

Quintero-Legorreta, O., 2002, Análisis estructural de fallas potencialmente activas: Boletín de la Sociedad Geológica Mexicana, 55(1), 12-29. https://doi.org/10.18268/ bsgm2002v55nlal

Rodríguez-Pérez,Q.,Zúñiga, F.R., 2017, Seismicity characterization of the Maravatío-Acambay and Actopan regions, central Mexico: Journal of South American Earth Sciences, 76, 264-275. https://doi.org/10.1016/j. jsames.2017.03.013

Singh,S.K.,Iglesias,A., Garduño, V. H., Quintanar, L., Ordaz, M., 2012, A source of the October, 2007 earthquake sequence of Morelia, Mexico and ground-motion estimation from larger earthquakes in the region: Geofisica Internacional, 51 (1), 73 - 86. https://doi.org/10.22201/ igeof.00167 169p.2012.51.1.147

Soria-Caballero, D.G., Garduño-Monroy, V.H., Alcala, M., Velázquez-Bucio, M., Grassi, L., 2019, Evidence for quaternary seismic activity of the La Alberca-Teremendo fault, Morelia region, Trans-Mexican Volcanic Belt: Revista Mexicana de Ciencias Geológicas, 36
(2), 242-258. https://doi.org/10.22201/ cgeo.20072902e.2019.2.1092

Stirling, M., Rhoades, D., Berryman, K., 2002, Comparison of Earthquake Scaling Relations Derived from Data of the Instrumental and Preinstrumental Era: Bulletin of the Seismological Society of America, 92 (2), 812 - 830. https://doi. org/10.1785/0120000221

Sunye-Puchol, I., Lacan, P., Ortuño, M., Villamor, P., Audin, L., Zúñiga, F. R., Langridge, R. M., Aguirre-Díaz, G. J., Lawton, T. F., 2015, La falla San Mateo: nuevas evidencias paleosismológicas de fallamiento activo en el graben de Acambay, México: Revista Mexicana de Ciencias Geológicas, 32 (3), 361-376.

Suter, M., Quintero-Legorreta, O. Johnson, C.A., 1992, Active faults and state of stress in the central part of the Trans-Mexican Volcanic Belt, Mexico; 1, The Venta de Bravo Fault: Journal of Geophysical Research. 97, 11983 - 11993. https:// doi.org/10.1029/91jb00428

Suter, M., 2002, Active Faults in the central part of the Trans-Mexican Volcanic Belt, Mexico. Compilation for the map of major active faults, western Hemisphere, International Lithosphere Program (ILP), Project II-2. December 1994, updated 2002. $137 \mathrm{p}$.

Suter,M.,López-Martínez,M.,QuinteroLegorreta,O.,Carrillo-Martínez,M., 2001,Quaternary intra-arc extension in the Central Trans-Mexican volcanic belt: Geological Society of America Bulletin,113(6),693-703. https://doi.org/10.1130/0016$7606(2001) 113<0693$ :qiaeit $>2.0$. со;2

Suter, M., 2015, The AD 1567 Mw 7.2 Ameca, Jalisco, Earthquake (Western TransMexican Volcanic Belt): Surface Rupture Parameters, Seismogeological Effects, and Macroseismic Intensities from Historical 
Sources: Bulletin of the Seismological Society of America, 105 (2A), 646-656. https://doi. org/10.1785/0120140163

Suter, M., 2016, Structure and Holocene rupture of the Morelia fault, Trans-Mexican volcanic belt, and their significance for seismic-hazard assessment: Bulletin of the Seismological Society of America, 106 (5), 2376-2388. https://doi.org/10.1785/0120160092

Urbina, F., Camacho, H., 1913, La zona megasísmica Acambay- Tixmadejé, Estado de México, conmovida el 19 de noviembre de 1912: Boletín del Instituto Geológico de México, 32, $125 \mathrm{p}$.

Velázquez-Bucio,M.M.,Garduño-Monroy, V.H., 2018, Soft-sediment deformation structures induced by seismic activity in the San Pedro el Alto area, Acambay graben, México: Revista Mexicana de Ciencias Geológicas, 35 (1), 28-40. https://doi.org/10.22201/ cgeo.20072902e.2018.1.530

Wells,D.L.,Coppersmith,K.J., 1994,New empirical relationships among magnitude, rupture length, rupture width, rupture area, and surface displacement: Bulletin of the Seismological Society of America, 84, 974 - 1002.

Wesnousky,S.G., 2008, Displacement and geometrical characteristics of earthquake surface ruptures: Issues and implications for seismic-hazard analysis and the process of earthquake rupture: Bulletin of the SeismologicalSociety of America, 98(4), 16091632. https://doi.org/10.1785/0120070111 


\section{ANEXO}

Tabla complementaria 1. Ubicación inicial y final de los perfiles topográficos de dirección NO - SE, trazados sobre el volcán El Picacho. Perfiles graficados en la figura 5.

\begin{tabular}{|c|c|c|c|c|c|c|c|c|c|}
\hline \multicolumn{10}{|c|}{ Dirección: $N O-S E$} \\
\hline \multirow[t]{2}{*}{ ID } & \multicolumn{2}{|c|}{ COORDENADAS } & \multicolumn{2}{|c|}{ ELEVACIÓN } & \multirow[t]{2}{*}{ ID } & \multicolumn{2}{|c|}{ COORDENADAS } & \multicolumn{2}{|c|}{ ELEVACIÓN } \\
\hline & INICIAL & FINAL & INICIAL & FINAL & & INICIAL & FINAL & INICIAL & FINAL \\
\hline $\mathbf{A}$ & $\begin{array}{l}0242342 \\
2196267\end{array}$ & $\begin{array}{l}0245848 \\
2189352\end{array}$ & 2106 & 2130 & $\mathbf{N}$ & $\begin{array}{l}0244723 \\
2197367\end{array}$ & $\begin{array}{l}0248309 \\
2190685\end{array}$ & 2203 & 2197 \\
\hline B & $\begin{array}{l}0242478 \\
2196325\end{array}$ & $\begin{array}{l}0246043 \\
2189468\end{array}$ & 2107 & 2130 & $\mathbf{O}$ & $\begin{array}{l}0244879 \\
2197426\end{array}$ & $\begin{array}{l}0248563 \\
2190783\end{array}$ & 2219 & 2200 \\
\hline C & $\begin{array}{l}0242634 \\
2196403\end{array}$ & $\begin{array}{l}0246199 \\
2189605\end{array}$ & 2111 & 2130 & $\mathbf{P}$ & $\begin{array}{l}0245075 \\
2197563\end{array}$ & $\begin{array}{l}0248740 \\
2190901\end{array}$ & 2247 & 2204 \\
\hline D & $\begin{array}{l}0242829 \\
2196442\end{array}$ & $\begin{array}{l}0246374 \\
2189722\end{array}$ & 2097 & 2130 & $\mathbf{Q}$ & $\begin{array}{l}0245212 \\
2197661\end{array}$ & $\begin{array}{l}0248994 \\
2191019\end{array}$ & 2272 & 2227 \\
\hline $\mathbf{E}$ & $\begin{array}{l}0242946 \\
2196501\end{array}$ & $\begin{array}{l}0246569 \\
2189858\end{array}$ & 2080 & 2137 & $\mathbf{R}$ & $\begin{array}{l}0245467 \\
2197701\end{array}$ & $\begin{array}{l}0249190 \\
2191136\end{array}$ & 2354 & 2236 \\
\hline $\mathbf{F}$ & $\begin{array}{l}0243180 \\
2196578\end{array}$ & $\begin{array}{l}0246764 \\
2189916\end{array}$ & 2090 & 2143 & $\mathbf{S}$ & $\begin{array}{l}0245702 \\
2197798\end{array}$ & $\begin{array}{l}0249347 \\
2191254\end{array}$ & 2405 & 2244 \\
\hline $\mathbf{G}$ & $\begin{array}{l}0243316 \\
2196676\end{array}$ & $\begin{array}{l}0246978 \\
2190033\end{array}$ & 2100 & 2146 & $\mathbf{T}$ & $\begin{array}{l}0245879 \\
2197896\end{array}$ & $\begin{array}{l}0249523 \\
2191391\end{array}$ & 2303 & 2264 \\
\hline $\mathbf{H}$ & $\begin{array}{l}0243550 \\
2196754\end{array}$ & $\begin{array}{l}0247154 \\
2190053\end{array}$ & 2120 & 2157 & $\mathbf{U}$ & $\begin{array}{l}0246094 \\
2197955\end{array}$ & $\begin{array}{l}0249719 \\
2191489\end{array}$ & 2246 & 2284 \\
\hline I & $\begin{array}{l}0243745 \\
2196851\end{array}$ & $\begin{array}{l}0247329 \\
2190209\end{array}$ & 2132 & 2171 & $\mathbf{V}$ & $\begin{array}{l}0246271 \\
2198014\end{array}$ & $\begin{array}{l}0249915 \\
2191587\end{array}$ & 2224 & 2341 \\
\hline $\mathbf{J}$ & $\begin{array}{l}0243900 \\
2196968\end{array}$ & $\begin{array}{l}0247543 \\
2190326\end{array}$ & 2145 & 2179 & W & $\begin{array}{l}0246486 \\
2198112\end{array}$ & $\begin{array}{l}0250072 \\
2191724\end{array}$ & 2211 & 2402 \\
\hline $\mathbf{K}$ & $\begin{array}{l}0244115 \\
2197085\end{array}$ & $\begin{array}{l}0247738 \\
2190423\end{array}$ & 2151 & 2192 & $\mathbf{X}$ & $\begin{array}{l}0246702 \\
2198171\end{array}$ & $\begin{array}{l}0250346 \\
2191802\end{array}$ & 2190 & 2331 \\
\hline $\mathbf{L}$ & $\begin{array}{l}0244290 \\
2197143\end{array}$ & $\begin{array}{l}0247874 \\
2190559\end{array}$ & 2165 & 2201 & $\mathbf{Y}$ & $\begin{array}{l}0246858 \\
2198288\end{array}$ & $\begin{array}{l}0250542 \\
2191940\end{array}$ & 2167 & 2270 \\
\hline M & $\begin{array}{l}0244468 \\
2197269\end{array}$ & $\begin{array}{l}0248073 \\
2190607\end{array}$ & 2183 & 2200 & $\mathbf{Z}$ & $\begin{array}{l}0247015 \\
2198426\end{array}$ & $\begin{array}{l}0250680 \\
2192194\end{array}$ & 2156 & 2270 \\
\hline
\end{tabular}

Tabla complementaria 2. Resumen de los parámetros medidos para la falla Malpaso - El Salto (FMAS) tanto recopilados como los obtenidos en este trabajo.

\begin{tabular}{|l|l|}
\hline PARÁMETRO & VALOR \\
\hline Espesor de la corteza sismogénica & $\begin{array}{l}12-20 \mathrm{~km} \text { (Ortuño et al., 2015) } \\
15 \mathrm{~km} \text { (Rodríguez-Pérez y Zúñiga, 2017) }\end{array}$ \\
\hline Rigidez de la corteza & $3 \times 10^{11} \mathrm{dyn} / \mathrm{cm}^{2}$ \\
\hline Longitud de ruptura superficial máxima (LRS $\left.{ }_{\max }\right)$ & $28.874 \mathrm{~km}$ \\
\hline Área de ruptura $(A)$ & $8.22 \mathrm{~km}^{2}$ \\
\hline Escarpe acumulado & $120 \mathrm{~m}( \pm 15 \mathrm{~m})$ \\
\hline Tasa de desplazamiento (SR) (Suter et al., 2001) & $0.16 \mathrm{~mm} / \mathrm{año}$ \\
\hline Tasa de desplazamiento estimada (este trabajo) & $0.086 \pm 0.015 \mathrm{~mm} / \mathrm{año}$ \\
\hline Período de recurrencia estimado (este trabajo) & $10021 \pm 1221$ años \\
\hline Magnitud potencial máxima $\left(M_{\mathrm{w}}\right)$ & $6.6-7.3$ \\
\hline
\end{tabular}

